\title{
FROM ATIYAH CLASSES TO HOMOTOPY LEIBNIZ ALGEBRAS
}

\author{
ZHUO CHEN, MATHIEU STIÉNON, AND PING XU
}

\begin{abstract}
A celebrated theorem of Kapranov states that the Atiyah class of the tangent bundle of a complex manifold $X$ makes $T_{X}[-1]$ into a Lie algebra object in $D^{+}(X)$, the bounded below derived category of coherent sheaves on $X$. Furthermore Kapranov proved that, for a Kähler manifold $X$, the Dolbeault resolution $\Omega^{\bullet-1}\left(T_{X}^{1,0}\right)$ of $T_{X}[-1]$ is an $L_{\infty}$ algebra. In this paper, we prove that Kapranov's theorem holds in much wider generality for vector bundles over Lie pairs. Given a Lie pair $(L, A)$, i.e. a Lie algebroid $L$ together with a Lie subalgebroid $A$, we define the Atiyah class $\alpha_{E}$ of an $A$-module $E$ as the obstruction to the existence of an $A$-compatible $L$-connection on $E$. We prove that the Atiyah classes $\alpha_{L / A}$ and $\alpha_{E}$ respectively make $L / A[-1]$ and $E[-1]$ into a Lie algebra and a Lie algebra module in the bounded below derived category $D^{+}(\mathcal{A})$, where $\mathcal{A}$ is the abelian category of left $\mathcal{U}(A)$-modules and $\mathcal{U}(A)$ is the universal enveloping algebra of $A$. Moreover, we produce a homotopy Leibniz algebra and a homotopy Leibniz module stemming from the Atiyah classes of $L / A$ and $E$, and inducing the aforesaid Lie structures in $D^{+}(\mathcal{A})$.
\end{abstract}

\section{Contents}

Introduction

Acknowledgments

1. Preliminaries: connections, modules, Lie pairs, and matched pairs

2. Atiyah classes

2.1. Prelude: holomorphic connections

2.2. Existence of $A$-compatible $L$-connections

2.3. Functoriality

2.4. Scalar Atiyah classes and Todd class

2.5. Jet short exact sequence

Research partially supported by NSFC grant 11471179, the Beijing high education young elite teacher project, NSA grant H98230-12-1-0234, and NSF grants DMS0605725, DMS0801129, DMS1101827. 


\section{INTRODUCTION}

The Atiyah class of a holomorphic vector bundle $E$ over a complex manifold $X$, as initially introduced by Atiyah [3], constitutes the obstruction to the existence of a holomorphic connection on said holomorphic vector bundle. It is constructed in the following way. The vector bundle $\mathscr{J}^{1} E$ of jets (of order 1) of holomorphic sections of $E \rightarrow X$ fits into the canonical short exact sequence

$$
0 \rightarrow T_{X}^{*} \otimes E \rightarrow \mathscr{J}^{1} E \rightarrow E \rightarrow 0
$$

of holomorphic vector bundles (over the complex manifold $X$ ). The Atiyah class of $E \rightarrow X$ is the extension class

$$
\alpha_{E} \in \operatorname{Ext}_{X}^{1}\left(E, T_{X}^{*} \otimes E\right) \cong H^{1}\left(X, T_{X}^{*} \otimes \operatorname{End} E\right)
$$

of this short exact sequence [3, 23].

In the late 1990's, Rozansky and Witten proposed a construction of a family of 3-dimensional topological quantum field theories, indexed by compact (or asymptotically flat) hyperKähler manifolds [46]. Thus, to each compact hyper-Kähler manifold, the RozanskyWitten procedure associates a topological invariant of 3-manifolds. In subsequent work, Kapranov [23] and Kontsevich [25] revealed the crucial role played by Atiyah classes in the construction of the Rozansky-Witten invariants. In particular, they showed that the hyper-Kähler restriction is unnecessary and that the theory devised by Rozansky and Witten works for all holomorphic symplectic manifolds [23, 25]. Kapranov's work highlighted a key fact: the Atiyah class of the tangent bundle of a complex manifold $X$ yields a morphism $T_{X}[-1] \otimes T_{X}[-1] \rightarrow T_{X}[-1]$ in the bounded below derived category $D^{+}(X)$ of coherent sheaves on $X$, which turns $T_{X}[-1]$ into a Lie algebra object in $D^{+}(X)$. Therefore, Kapranov's approach shone light on many similarities between the Rozansky-Witten and Chern-Simons theories [4, 5] as stressed by Roberts and Willerton [45].

Atiyah classes have also enjoyed renewed vigor due to Kontsevich's seminal work on deformation quantization [26, 24]. Kontsevich indicated the existence of deep ties between the Todd genus of complex manifolds and the Duflo element in Lie theory [26, 24, 47, 11]. This discovery inspired several subsequent works on Hochschild (co)homology and the Hochschild-Kostant-Rosenberg isomorphism for complex manifolds, by Dolgushev, Tarmarkin \& Tsygan [18, 17], Căldăraru [16], Markarian [38], Ramadoss [43], and Calaque \& Van den Bergh [12] among many others. In particular, the work of Markarian [38] (see also Ramadoss [43]) led to an alternative proof of the Hirzebruch-Riemann-Roch theorem and its variations.

In [41, 42, Molino introduced an Atiyah class for connections "transverse to a foliation," which measures the obstruction to their "projectability." Molino's class has applications in geometry, for instance, in the study of differential operators on foliated manifolds [50] and in deformation quantization [6].

This paper is the first in a sequence of works [13, 31, 32, 54, 39] which aim at developing in a general setting a theory of Atiyah classes and their applications. Our goal is to explore emerging connections between derived geometry and classical areas of mathematics such as complex geometry, foliation theory, Poisson geometry and Lie theory. The present paper develops a framework which encompasses both the original Atiyah class of holomorphic vector bundles and the Molino class of real vector bundles foliated over a foliation as special cases.

Lie algebroids are the starting point of our approach. Indeed, holomorphic vector bundles and vector bundles foliated over a foliation may both be seen as instances of the concept of module over a Lie algebroid, a straightforward generalization of the well-known representations of Lie algebras. Given a Lie algebroid $L$ over a base manifold $M$ with 
anchor $\rho: L \rightarrow T M$, an $L$-connection on a vector bundle $E \rightarrow M$ is a bilinear map $X \otimes s \mapsto \nabla_{X} s$ from $\Gamma(L) \otimes \Gamma(E)$ to $\Gamma(E)$ satisfying the usual axioms: $\nabla_{f X} s=f \nabla_{X} s$ and $\nabla_{X}(f s)=\rho(X) f \cdot s+f \nabla_{X} s$ for all $f \in C^{\infty}(M)$. If the connection is flat, $E$ is said to be a module over the Lie algebroid $L$. When the base is the one-point space, the $L$-modules are simply Lie algebra modules in the classical sense. When the base is a complex manifold $X$, the holomorphic vector bundles over $X$ are equivalent to the modules of the complex Lie algebroid $T_{X}^{0,1}$ stemming from the complex manifold $X$. Molino's foliated bundles are modules over the Lie algebroid structure corresponding to the foliation of their base.

We introduce a general theory of Atiyah classes of vector bundles over Lie algebroid pairs. By a Lie algebroid pair $(L, A)$, we mean a Lie algebroid $L$ (over a manifold $M$ ) together with a Lie subalgebroid $A$ (over the same base $M$ ) of $L$. And by a vector bundle $E$ over the Lie algebroid pair $(L, A)$, we mean a vector bundle $E$ (over $M$ ), which is a module over the Lie subalgebroid $A$. Given such a Lie algebroid pair $(L, A)$ and $A$-module $E$, we consider the jet bundle $\mathscr{J}_{L / A}^{1} E$ (of order 1 ), whose smooth sections are the $L$-connections on $E$ extending the (infinitesimal) $A$-action on $E$ in a compatible way. We prove the following

Theorem A. The jet bundle $\mathscr{J}_{L / A}^{1} E$ is naturally an A-module. It fits in a short exact sequence of $A$-modules

$$
0 \rightarrow A^{\perp} \otimes E \rightarrow \mathscr{J}_{L / A}^{1} E \rightarrow E \rightarrow 0 .
$$

Here $A^{\perp}$ denotes the annihilator of $A$ in $L$.

We call the extension class

$$
\alpha_{E} \in \operatorname{Ext}_{\mathcal{A}}^{1}\left(E, A^{\perp} \otimes E\right) \cong H^{1}\left(A, A^{\perp} \otimes \operatorname{End} E\right)
$$

of this short exact sequence, the Atiyah class of $E$ because, when $L=T_{X} \otimes \mathbb{C}$ and $A=T_{X}^{0,1}$ for a complex manifold $X, \mathscr{J}_{L / A}^{1} E$ is the space of 1-jets of holomorphic sections of $E \rightarrow X$ and $\alpha_{E}$ is the (classical) Atiyah class of $E \rightarrow X$; and, when $L$ is the tangent bundle of a smooth manifold $M$ and $A$ is an integrable distribution on $M, \alpha_{E}$ is the Molino class of the vector bundle $E$ foliated over $A$. Geometrically, the Atiyah class can thus be interpreted as the obstruction to the existence of a compatible $L$-connection on $E$ which extends the $A$-action with which $E$ is endowed.

It turns out that the Atiyah class introduced in our general context and the classical Atiyah class of holomorphic vector bundles enjoy similar algebraic properties.

We denote the abelian category of modules over the universal enveloping algebra $\mathcal{U}(A)$ of the Lie algebroid $A$ by the symbol $\mathcal{A}$. Every vector bundle over $M$ endowed with an $A$-action - more precisely its space of smooth sections - is an object of $\mathcal{A}$. The bounded below derived category of $\mathcal{A}$ will be denoted by $D^{+}(\mathcal{A})$.

Given a Lie algebroid pair $(L, A)$, the quotient $L / A$ is naturally an $A$-module. When $L$ is the tangent bundle to a manifold $M$ and $A$ is an integrable distribution on $M$, the $A$-action on $L / A$ is given by the Bott connection [8]. We consider $L / A$ as a complex concentrated in degree 1 and refer to it as $L / A[-1]$. We show that the Atiyah class of $L / A$ makes $L / A[-1]$ into a Lie algebra object in the derived category $D^{+}(\mathcal{A})$. Indeed, being an element of

$$
\begin{aligned}
\operatorname{Ext}_{\mathcal{A}}^{1}\left(L / A, A^{\perp} \otimes L / A\right) & \cong \operatorname{Ext}_{\mathcal{A}}^{1}(L / A \otimes L / A, L / A) \\
& \cong \operatorname{Hom}_{D^{+}(\mathcal{A})}(L / A \otimes L / A, L / A[1]) \\
& \cong \operatorname{Hom}_{D^{+}(\mathcal{A})}(L / A[-1] \otimes L / A[-1], L / A[-1]),
\end{aligned}
$$

the Atiyah class $\alpha_{L / A}$ of the $A$-module $L / A$ defines a "Lie bracket" on $L / A[-1]$. Moreover, if $E$ is an $A$-module, its Atiyah class

$$
\alpha_{E} \in \operatorname{Ext}_{\mathcal{A}}^{1}\left(E, A^{\perp} \otimes E\right) \cong \operatorname{Ext}_{\mathcal{A}}^{1}(L / A \otimes E, E) \cong \operatorname{Hom}_{D^{+}(\mathcal{A})}(L / A[-1] \otimes E[-1], E[-1])
$$


defines a "representation" on $E[-1]$ of the "Lie algebra" $L / A[-1]$. In summary, we prove the following

Theorem B. Let $(L, A)$ be a Lie algebroid pair with quotient $L / A$. Then the Atiyah class of $L / A$ turns $L / A[-1]$ into a Lie algebra object in the derived category $D^{+}(\mathcal{A})$. Moreover, if $E$ is an $A$-module, then $E[-1]$ is a module object over the Lie algebra object $L / A[-1]$ in the derived category $D^{+}(\mathcal{A})$.

The above result suggests that, on the cochain level, the Atiyah class should define some kind of Lie algebra up to homotopy. But how does one obtain a cocycle representing the Atiyah class? Recall that a Dolbeault representative of the Atiyah class of a holomorphic vector bundle $E \rightarrow X$ can be obtained in the following way. Considering $T_{X}^{1,0}$ as a complex Lie algebroid, choose a $T_{X}^{1,0}$-connection $\nabla^{1,0}$ on $E$. Being a holomorphic vector bundle, $E$ carries a canonical flat $T_{X}^{0,1}$-connection $\bar{\partial}$. Adding $\nabla^{1,0}$ and $\bar{\partial}$, we obtain a $T_{X} \otimes \mathbb{C}$-connection $\nabla$ on $E$. The element $\mathcal{R} \in \Omega^{1,1}$ (End $E$ ) defined by $\mathcal{R}\left(X^{0,1}, Y^{1,0}\right) s=\nabla_{X^{0,1}} \nabla_{Y^{1,0}} s-\nabla_{Y^{1,0}} \nabla_{X^{0,1}} s-\nabla_{\left[X^{0,1}, Y^{1,0}\right]} s$ is a Dolbeault 1-cocycle whose cohomology class (which is independent of the choice of $\nabla^{1,0}$ ) is the Atiyah class $\alpha_{E} \in H^{1,1}(X$, End $E) \cong H^{1}\left(X, T_{X}^{*} \otimes\right.$ End $\left.E\right)$. In the more general setting of vector bundles over Lie algebroid pairs, the Atiyah class can be recovered from a Lie algebroid connection as follows. Assume $(L, A)$ is a Lie pair, and $E$ is an $A$-module. Let $\nabla$ be any $L$-connection on $E$ extending its $A$-action. The curvature of $\nabla$ is the bundle map $R^{\nabla}: \wedge^{2} L \rightarrow$ End $E$ defined by $R^{\nabla}\left(l_{1}, l_{2}\right)=\nabla_{l_{1}} \nabla_{l_{2}}-\nabla_{l_{2}} \nabla_{l_{1}}-\nabla_{\left[l_{1}, l_{2}\right]}$, for all $l_{1}, l_{2} \in \Gamma(L)$. Since $E$ is an $A$-module, the restriction of $R^{\nabla}$ to $\wedge^{2} A$ vanishes. Hence the curvature determines a section $R_{E}^{\nabla} \in \Gamma\left(A^{*} \otimes A^{\perp} \otimes\right.$ End $\left.E\right)$, which happens to be a 1-cocycle for the Lie algebroid $A$ with values in the $A$-module $A^{\perp} \otimes$ End $E$. We prove that the cohomology class $\alpha_{E} \in H^{1}\left(A, A^{\perp} \otimes\right.$ End $\left.E\right)$ of the 1-cocycle $R_{E}^{\nabla}$ is the Atiyah class of the $A$-module $E$.

When the $A$-module $E$ is the quotient $L / A$ of the Lie algebroid pair $(L, A)$, the choice of an $L$-connection $\nabla$ on $L / A$ extending the $A$-action, yields the Atiyah 1-cocycle $R_{L / A}^{\nabla} \in$ $\Gamma\left(A^{*} \otimes(L / A)^{*} \otimes \operatorname{End}(L / A)\right)$, which may be regarded as a bundle map $R_{2}: L / A \otimes L / A \rightarrow$ $A^{*} \otimes L / A$. Starting from $R_{2}$ and a splitting of the short exact sequence of vector bundles $0 \rightarrow A \rightarrow L \rightarrow L / A \rightarrow 0$, a sequence $\left(R_{n}\right)_{n=2}^{\infty}$ of bundle maps

$$
R_{n}: \otimes^{n} L / A \rightarrow \operatorname{Hom}(A, L / A)
$$

can be defined inductively by the relation $R_{n+1}=\partial^{\nabla} R_{n}$, for $n \geq 2$. Alternatively, $R_{n}$ can be seen as a section of the vector bundle $A^{*} \otimes\left(\otimes^{n}(L / A)^{*}\right) \otimes L / A$. Then the graded vector space $V=\bigoplus_{n=0}^{\infty} \Gamma\left(\wedge^{n} A^{*} \otimes L / A\right)$ can be endowed with a sequence $\left(\lambda_{k}\right)_{k \in \mathbb{N}}$ of multibrackets $\lambda_{k}: \otimes^{k} V \rightarrow V$ : the unary bracket $\lambda_{1}$ is chosen to be the coboundary operator $\partial^{A}$ of exterior forms on the Lie algebroid $A$ taking values in the $A$-module $L / A$, while all higher order brackets $\lambda_{k}$ are defined by the relation

$$
\lambda_{k}\left(\xi_{1} \otimes b_{1}, \cdots, \xi_{k} \otimes b_{k}\right)=(-1)^{\left|\xi_{1}\right|+\cdots+\left|\xi_{k}\right|} \xi_{1} \wedge \cdots \wedge \xi_{k} \wedge R_{k}\left(b_{1}, \cdots, b_{k}\right),
$$

where $b_{1}, \ldots, b_{k} \in \Gamma(L / A)$ and $\xi_{1}, \ldots, \xi_{k}$ are arbitrary homogeneous elements of $\Gamma\left(\wedge^{\bullet} A^{*}\right)$.

By an $A$-algebra, we mean a bundle of associative algebras $\mathcal{C}$ over $M$ endowed with an $A$-module structure such that $\Gamma(A)$ acts by derivations. For a commutative $A$-algebra $\mathcal{C}$, $\left(\lambda_{k}\right)_{k=1}^{\infty}$ extends in a natural way to the graded vector space $\bigoplus_{n=0}^{\infty} \Gamma\left(\wedge^{n} A^{*} \otimes L / A \otimes \mathcal{C}\right)$. We prove

Theorem C. Assume that $(L, A)$ is a Lie pair and $\mathcal{C}$ is a commutative A-algebra. When endowed with the sequence of multibrackets $\left(\lambda_{k}\right)_{k \in \mathbb{N}}$, the graded vector space $\Gamma\left(\wedge^{\bullet} A^{*} \otimes\right.$ $L / A \otimes \mathcal{C})[-1]$ becomes a Leibniz ${ }_{\infty}$ algebra - a natural generalization of Stasheff's $L_{\infty}$ 
algebras [29] first introduced by Loday [34] in which the requirement that the multibrackets be (skew-)symmetric is dropped.

If $E$ is an $A$-module, the graded vector space $\Gamma\left(\wedge^{\bullet} A^{*} \otimes E \otimes \mathcal{C}\right)[-1]$ becomes a Leibniz $z_{\infty}$ module over the Leibniz $\infty$ algebra $\Gamma\left(\wedge^{\bullet} A^{*} \otimes L / A \otimes \mathcal{C}\right)[-1]$.

As a consequence, $\bigoplus_{i \geq 1} H^{i-1}(A, L / A \otimes \mathcal{C})$ is a graded Lie algebra and $\bigoplus_{i \geq 1} H^{i-1}(A, E \otimes \mathcal{C})$ a module over it.

We also identify a simple criterion for detecting when this Leibniz algebra is actually an $L_{\infty}$ algebra. This criterion being satisfied when $X$ is a Kähler manifold, $L=T_{X} \otimes \mathbb{C}$ and $A=T_{X}^{0,1}$, we recover the $L_{\infty}$-structure on $\Omega^{0, \bullet-1}\left(T^{1,0}\right)$ discovered by Kapranov [23].

Recently, we proved that the sequence of multibrackets $\left\{\lambda_{k}\right\}_{k \in \mathbb{N}}$ of Theorem C can be tweaked so as to make $\Gamma\left(\wedge^{\bullet} A^{*} \otimes L / A\right)$ an $L_{\infty}[1]$ algebra rather than a mere Leibniz ${ }_{\infty}[1]$ algebra [31, 32]. Such an $L_{\infty}$ [1] algebra should be related to the $L_{\infty}$-spaces of Costello [14, 15]. The intrinsic meaning of this homotopy algebraic structure arising from the Atiyah class of a Lie algebroid pairs is explored in [31, 32, 33. Our definition of the Atiyah class can also be extended to complexes of $A$-modules [13] as was done in [38] for complexes of coherent sheaves of $\mathcal{O}_{X}$-modules. The universal enveloping algebra of the Lie algebra object $L / A[-1]$ in the derived category $D^{+}(\mathcal{A})$ (see Theorem $B$ ) is described in that same paper [13. We note that the Atiyah class of Lie algebroid pairs plays a central role in the construction of new Rozansky-Witten type invariants of 3-manifolds from symplectic Lie pairs [54]. In another direction, Atiyah classes were defined for differential graded vector bundles and these yield homotopy algebraic structures as well [39].

We also would like to point out works of others which are related to the present paper. Vitagliano studied various homotopy algebra structures associated to foliations (a special case of Lie algebroid pairs) [51, 53, 52. For the Atiyah class of a DG-module over a dDG-algebra, the reader will want to consult Calaque's work [12]. After the first draft of this paper was posted on arXiv, Calaque inferred that, for Lie algebra pairs $(\mathfrak{d}, \mathfrak{g})$, i.e. Lie algebroid pairs with the one-point space as base manifold, the Atiyah class of the quotient $\mathfrak{d} / \mathfrak{g}$ coincides with the class capturing the obstruction to the "PBW problem" studied earlier by Calaque-Căldăraru-Tu [10] (see also [19]). Bordemann gave a nice interpretation of the Calaque-Căldăraru-Tu class as the obstruction to the existence of invariant connections on homogeneous spaces [7]. Another recent development is Calaque's beautiful work [9] on the relation between the Atiyah class of the $A$-module $L / A$ with respect to the Lie pair $(L, A)$ and the relative PBW problem previously solved by Căldăraru-Calaque-Tu [10]. For more on this topic, we also refer the reader to work of Laurent-Gengoux \& Voglaire [33]. Calaque also pointed out to us that our results should be related to the obstruction to a relative Hochschild-Kostant-Rosenberg isomorphism for closed embeddings of algebraic varieties identified by Arinkin \& Căldăraru [2]. This certainly deserves further investigation. Finally, we would like to mention, in relation to the homotopy algebra results of the present paper, Yu's work on $L_{\infty}$-algebroids [56]. Stasheff's work on constrained Poisson algebras [48] is another interesting result which could well be related to the present paper.

Acknowledgments. We would like to express our gratitude to several institutions for their hospitality while we were working on this project: Penn State University (Chen), Université Paris 7 (Stiénon), Université du Luxembourg (Chen and Stiénon), Institut des Hautes Études Scientifiques (Xu), and Beijing International Center for Mathematical Research $(\mathrm{Xu})$. We would also like to thank Martin Bordemann, Paul Bressler, Damien Calaque, Murray Gerstenhaber, Grégory Ginot, Camille Laurent-Gengoux, Boris Shoikhet, Jim Stasheff, Izu Vaisman and Alan Weinstein for fruitful discussions and useful comments. Special thanks go to Jim Stasheff for carefully reading the preliminary version of the manuscript. 


\section{Preliminaries: Connections, modules, Lie pairs, And matched pairs}

Let $M$ be a smooth manifold, let $L \rightarrow M$ be a Lie algebroid, and let $E \stackrel{\pi}{\rightarrow} M$ be a vector bundle. The anchor map of $L$ is denoted by $\rho$.

Recall that the Lie algebroid differential $d: \Gamma\left(\wedge^{\bullet} L^{*}\right) \rightarrow \Gamma\left(\wedge^{\bullet+1} L^{*}\right)$ is given by

$$
\begin{aligned}
&(d \mu)\left(x_{0}, \cdots, x_{n}\right)= \sum_{i=0}^{n}(-1)^{i} \rho\left(x_{i}\right)\left(\mu\left(x_{0}, \cdots, \widehat{x_{i}}, \cdots, x_{n}\right)\right) \\
&+\sum_{i<j}(-1)^{i+j} \mu\left(\left[x_{i}, x_{j}\right], x_{0}, \cdots, \widehat{x_{i}}, \cdots, \widehat{x_{j}}, \cdots, x_{n}\right), \\
& \forall \mu \in \Gamma\left(\wedge^{n} L^{*}\right), x_{i} \in \Gamma(L), i=0, \cdots, n .
\end{aligned}
$$

The traditional description of a (linear) $L$-connection on $E$ is in terms of a covariant derivative

$$
\Gamma(L) \times \Gamma(E) \rightarrow \Gamma(E):(x, e) \mapsto \nabla_{x} e
$$

characterized by the following two properties:

$$
\begin{gathered}
\nabla_{f x} e=f \nabla_{x} e, \\
\nabla_{x}(f e)=\rho(x) f \cdot e+f \cdot \nabla_{x} e,
\end{gathered}
$$

for all $x \in \Gamma(L), e \in \Gamma(E)$, and $f \in C^{\infty}(M)$.

Here, we give three equivalent descriptions of (linear) $L$-connections on $E$ : covariant differential, horizontal lifting, and horizontal distribution.

Definition 1.1. A (linear) L-connection on $E$ is a map $\Gamma(E) \stackrel{d^{\nabla}}{\longrightarrow} \Gamma\left(L^{*} \otimes E\right)$, called covariant differential, satisfying the Leibniz rule

$$
d^{\nabla}(f e)=\rho^{*}(d f) \otimes e+f \cdot d^{\nabla} e,
$$

for all $f \in C^{\infty}(M)$ and $e \in \Gamma(E)$.

The covariant differential $\Gamma(E) \stackrel{d^{\nabla}}{\longrightarrow} \Gamma\left(L^{*} \otimes E\right)$ extends uniquely to a degree 1 operator

$$
\Gamma\left(\wedge^{\bullet} L^{*} \otimes E\right) \stackrel{d^{\nabla}}{\longrightarrow} \Gamma\left(\wedge^{\bullet+1} L^{*} \otimes E\right)
$$

satisfying the Leibniz rule

$$
d^{\nabla}(\beta \otimes e)=d \beta \otimes e+(-1)^{b} \beta \otimes d^{\nabla} e,
$$

for all $\beta \in \Gamma\left(\wedge^{b} L^{*}\right)$ and $e \in \Gamma(E)$.

Definition 1.2. A (linear) L-connection on $E$ is a map $L \times_{M} E \stackrel{h}{\rightarrow} T_{E}$, called horizontal lifting, such that the diagram

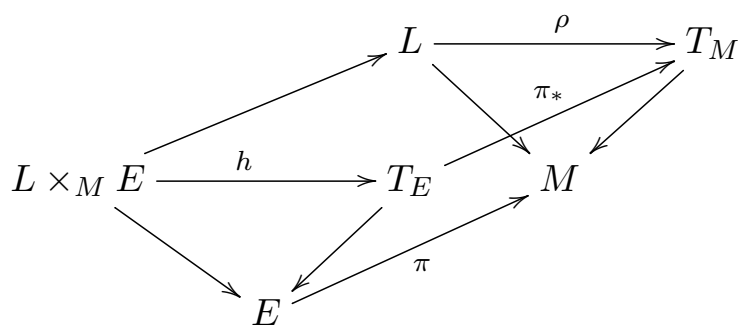


commutes and its faces
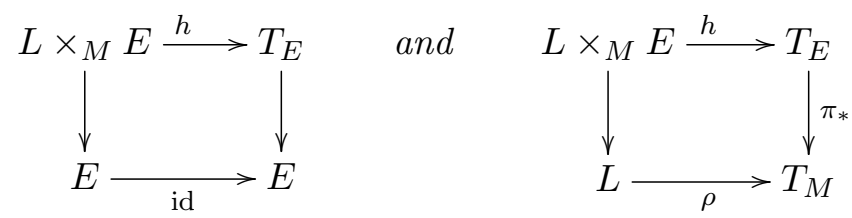

are vector bundle maps.

A vector field $X$ on $E$ is said to be projectable onto $M$ if $\pi\left(e_{1}\right)=\pi\left(e_{2}\right)$ implies $\pi_{*}\left(X_{e_{1}}\right)=$ $\pi_{*}\left(X_{e_{2}}\right)$. By $\mathfrak{X}_{\pi}(E)$, we denote the space of vector fields $X$ on $E$ which are projectable onto $M$ and whose flow $\Phi_{t}^{X}: E \rightarrow E$ is a vector bundle automorphism over the flow $\Phi_{t}^{\pi_{*} X}: M \rightarrow M$ of the projected vector field $\pi_{*} X$ on $M$. These vector fields are normally called linear vector fields on $E$ (see [37] for details). The space $\mathfrak{X}_{\pi}(E)$ of linear vector fields on $E$ is obviously a module over the ring $C^{\infty}(M)$.

Definition 1.3. A (linear) L-connection on $E$ is a morphism of $C^{\infty}(M)$-modules $\Gamma(L) \stackrel{H}{\rightarrow}$ $\mathfrak{X}_{\pi}(E)$, called horizontal distribution, such that the diagram

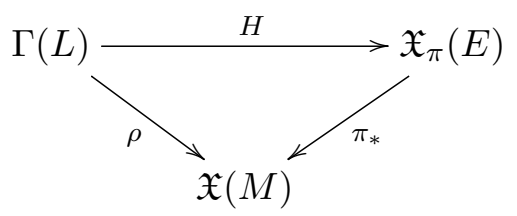

commutes.

Covariant differential, covariant derivative, horizontal lift, and horizontal distribution are related to one another by the identities

$$
\begin{gathered}
\nabla_{l} e=\left\langle d^{\nabla} e, l\right\rangle ; \\
e_{*} \rho\left(l_{x}\right)-h\left(l_{x}, e_{x}\right)=\tau_{e_{x}}\left(\nabla_{l} e\right)_{x} ; \\
\left.H(l)\right|_{e_{x}}=h\left(l_{x}, e_{x}\right),
\end{gathered}
$$

$\forall x \in M, l \in \Gamma(L)$, and $e \in \Gamma(E)$. Here in the second equation, $\tau_{e_{x}}$ denotes the canonical isomorphism between the fiber $E_{x}$ and its tangent space at the point $e_{x}$. This second equation can be rewritten as

$$
h\left(l_{x}, e_{x}\right) f_{\nu}=\left\langle\nabla_{l_{x}} \nu, e_{x}\right\rangle,
$$

where $f_{\nu}$ denotes the fiberwise linear function on $E$ determined by $\nu \in \Gamma\left(E^{*}\right)$.

The following assertions are equivalent:

$$
\begin{aligned}
& \nabla_{l_{1}} \nabla_{l_{2}}-\nabla_{l_{2}} \nabla_{l_{1}}=\nabla_{\left[l_{1}, l_{2}\right]} ; \\
& H\left(\left[l_{1}, l_{2}\right]\right)=\left[H\left(l_{1}\right), H\left(l_{2}\right)\right] .
\end{aligned}
$$

When they are satisfied for all $l_{1}, l_{2} \in \Gamma(L)$, the connection is said to be flat. An $L$-module is a vector bundle $E \rightarrow M$ endowed with a flat (linear) $L$-connection. A flat (linear) $L$ connection will also be called an $L$-action or $L$-representation. When the $L$-connection on $E$ is flat, $\left(d^{\nabla}\right)^{2}=0$ and $\left(\Gamma\left(\wedge^{\bullet} L \otimes E\right), d^{\nabla}\right)$ is a cochain complex, whose cohomology groups $H^{\bullet}(L, E)$ are the so-called Lie algebroid cohomology groups of $L$ with values in $E$.

By a Lie algebroid pair, or simply a Lie pair $(L, A)$, we mean a Lie algebroid $L$ and a Lie subalgebroid $A$ of $L$ over the same base manifold $M$. 
Proposition 1.4. The quotient $L / A$ of a Lie pair $(L, A)$ is an $A$-module; the action of $A$ on $L / A$ is defined by

$$
\nabla_{a}(q(l))=q([a, l]), \quad \forall a \in \Gamma(A), l \in \Gamma(L),
$$

where $q$ denotes the projection $L \rightarrow L / A$. Being dual to $L / A$, the annihilator $A^{\perp}$ of $A$ in $L^{*}$ is also an $A$-module.

Assume now that $A$ and $B$ are two Lie subalgebroids of a Lie algebroid $L$ such that $L$ and $A \oplus B$ are isomorphic as vector bundles. Then $L / A \cong B$ is naturally an $A$-module while $L / B \cong A$ is naturally a $B$-module. The Lie algebroids $A$ and $B$ are said to form a matched pair.

Definition 1.5 ([35, 40, 36]). Two (real or complex) Lie algebroids $A$ and $B$ over the same base manifold $M$ and with respective anchors $\rho_{A}$ and $\rho_{B}$ are said to form a matched pair if there exists an action $\nabla$ of $A$ on $B$ and an action $\Delta$ of $B$ on $A$ such that the identities

$$
\begin{gathered}
{\left[\rho_{A}(X), \rho_{B}(Y)\right]=-\rho_{A}\left(\Delta_{Y} X\right)+\rho_{B}\left(\nabla_{X} Y\right)} \\
\nabla_{X}\left[Y_{1}, Y_{2}\right]=\left[\nabla_{X} Y_{1}, Y_{2}\right]+\left[Y_{1}, \nabla_{X} Y_{2}\right]+\nabla_{\Delta_{Y_{2}} X} Y_{1}-\nabla_{\Delta_{Y_{1}} X} Y_{2}, \\
\Delta_{Y}\left[X_{1}, X_{2}\right]=\left[\Delta_{Y} X_{1}, X_{2}\right]+\left[X_{1}, \Delta_{Y} X_{2}\right]+\Delta_{\nabla_{X_{2}} Y} X_{1}-\Delta_{\nabla_{X_{1}} Y} X_{2},
\end{gathered}
$$

hold for all $X_{1}, X_{2}, X \in \Gamma(A)$ and $Y_{1}, Y_{2}, Y \in \Gamma(B)$.

Proposition 1.6 (40, 36]). Given a matched pair $(A, B)$ of Lie algebroids, there is a Lie algebroid structure $A \bowtie B$ on the direct sum vector bundle $A \oplus B$, with anchor

$$
X \oplus Y \mapsto \rho_{A}(X)+\rho_{B}(Y)
$$

and bracket

$$
\left[X_{1} \oplus Y_{1}, X_{2} \oplus Y_{2}\right]=\left(\left[X_{1}, X_{2}\right]+\Delta_{Y_{1}} X_{2}-\Delta_{Y_{2}} X_{1}\right) \oplus\left(\left[Y_{1}, Y_{2}\right]+\nabla_{X_{1}} Y_{2}-\nabla_{X_{2}} Y_{1}\right) .
$$

Conversely, if $A \oplus B$ has a Lie algebroid structure for which $A \oplus 0$ and $0 \oplus B$ are Lie subalgebroids, then the representations $\nabla$ and $\Delta$ defined by

$$
[X \oplus 0,0 \oplus Y]=-\Delta_{Y} X \oplus \nabla_{X} Y
$$

endow the couple $(A, B)$ with a structure of matched pair.

Example 1.7. A Lie algebra is a Lie algebroid whose base manifold is the one-point space. If the direct sum $\mathfrak{g} \oplus \mathfrak{g}^{*}$ of a vector space $\mathfrak{g}$ and its dual $\mathfrak{g}^{*}$ is endowed with a Lie algebra structure such that the direct summands $\mathfrak{g}$ and $\mathfrak{g}^{*}$ are Lie subalgebras and

$$
[X, \alpha]=\operatorname{ad}_{X}^{*} \alpha-\operatorname{ad}_{\alpha}^{*} X, \quad \forall X \in \mathfrak{g}, \alpha \in \mathfrak{g}^{*},
$$

the pair $\left(\mathfrak{g}, \mathfrak{g}^{*}\right)$ is said to be a Lie bialgebra. Lie bialgebras are instances of matched pairs of Lie algebroids.

Example 1.8. Let $X$ be a complex manifold. Then $\left(T_{X}^{0,1}, T_{X}^{1,0}\right)$ is a matched pair, where the actions are given by

$$
\nabla_{X^{0,1}} X^{1,0}=\operatorname{pr}^{1,0}\left[X^{0,1}, X^{1,0}\right] \quad \text { and } \quad \Delta_{X^{1,0}} X^{0,1}=\operatorname{pr}^{0,1}\left[X^{1,0}, X^{0,1}\right],
$$

for all $X^{0,1} \in \Gamma\left(T_{X}^{0,1}\right)$ and $X^{1,0} \in \Gamma\left(T_{X}^{1,0}\right)$. Hence $T_{X}^{0,1} \bowtie T_{X}^{1,0}$ and $T_{X} \otimes \mathbb{C}$ are isomorphic as complex Lie algebroids. More generally, given a holomorphic Lie algebroid $A$, the pair $\left(A^{0,1}, A^{1,0}\right)$ is a matched pair of Lie algebroids and $A^{0,1} \bowtie A^{1,0}$ is isomorphic, as a complex Lie algebroid, to $A \otimes \mathbb{C}[30$.

Example 1.9. Let $D$ be an integrable distribution on a smooth manifold $M$. Then $D$ is a Lie subalgebroid of $T_{X}$, and the normal bundle $T_{X} / D$ is canonically a $D$-module. The $D$-action on $T_{X} / D$ is usually called Bott connection [8]. Moreover, if $\mathcal{F}_{1}$ and $\mathcal{F}_{2}$ are two transversal foliations on a smooth manifold $M$, the corresponding tangent distributions $T_{\mathcal{F}_{1}}$ and $T_{\mathcal{F}_{2}}$ constitute a matched pair of Lie algebroids with $T_{\mathcal{F}_{1}} \bowtie T_{\mathcal{F}_{2}} \cong T_{X}$. 
Example 1.10. Let $G$ be a Poisson Lie group and let $(P, \pi)$ be a Poisson $G$-space, i.e. a Poisson manifold $P$ together with a $G$-action such that the action map $G \times P \rightarrow P$ is a Poisson map. According to $\mathrm{Lu}$ [35], $A=\left(T^{*} P\right)_{\pi}$ and $B=P \rtimes \mathfrak{g}$ form a matched pair of Lie algebroids.

Remark 1.11. A matched pair of Lie algebroids $L=A \bowtie B$ can be seen as a Lie pair $(L, A)$ together with a splitting $j: B \rightarrow L$ of the short exact sequence $0 \rightarrow A \rightarrow L \rightarrow B \rightarrow$ 0 , whose image $j(B)$ happens to be a Lie subalgebroid of $L$.

\section{Atiyah CLASSES}

2.1. Prelude: holomorphic connections. The Atiyah class of a holomorphic vector bundle $E$ over a complex manifold $X$ is the obstruction class to the existence of a holomorphic (linear) connection. It is constructed in the following way. The vector bundle $\mathscr{J}^{1} E$ of jets (of order 1 ) of holomorphic sections of $E \rightarrow X$ fits into the canonical short exact sequence of holomorphic vector bundles

$$
0 \rightarrow T_{X}^{*} \otimes E \rightarrow \mathscr{J}^{1} E \rightarrow E \rightarrow 0
$$

over the complex manifold $X$. The Atiyah class of $E \rightarrow X$ is the extension class

$$
\alpha_{E} \in \operatorname{Ext}_{X}^{1}\left(E, T_{X}^{*} \otimes E\right)
$$

of this short exact sequence [3, 23].

There are canonical isomorphisms between the abelian groups $\operatorname{Ext}_{X}^{1}\left(E, T_{X}^{*} \otimes E\right)$ and $\operatorname{Hom}_{D^{+}(X)}\left(T_{X} \otimes E, E[1]\right)$, the sheaf cohomology group $H^{1}\left(X, T_{X}^{*} \otimes\right.$ End $\left.E\right)$ and the Dolbeault cohomology group $H^{1,1}(X$, End $E)$. A Dolbeault representative of the Atiyah class can be obtained in the following way. Considering $T_{X}^{1,0}$ as a complex Lie algebroid, choose a $T_{X}^{1,0}$-connection $\nabla^{1,0}$ on $E$. Being a holomorphic vector bundle, $E$ carries a canonical flat $T_{X}^{0,1}$-connection $\bar{\partial}$. Adding $\nabla^{1,0}$ and $\bar{\partial}$, we obtain a $T_{X} \otimes \mathbb{C}$-connection $\nabla$ on $E$. The element $\mathcal{R} \in \Omega^{1,1}$ (End $E$ ) defined by

$$
\mathcal{R}\left(X^{0,1}, Y^{1,0}\right) s=\nabla_{X^{0,1}} \nabla_{Y^{1,0}} s-\nabla_{Y^{1,0}} \nabla_{X^{0,1}} s-\nabla_{\left[X^{0,1}, Y^{1,0}\right]} s
$$

is a Dolbeault 1-cocycle whose cohomology class (which is independent of the choice of $\left.\nabla^{1,0}\right)$ is the Atiyah class $\alpha_{E} \in H^{1,1}(X$, End $E)$.

\subsection{Existence of $A$-compatible $L$-connections.}

2.2.1. Extension of an A-action to a compatible L-connection. Throughout this section, $(L, A)$ is a Lie pair and $E$ is an $A$-module. The symbols $\mathscr{E}$ and $\mathscr{L}$ will denote the sheaves on $M$ defined by

$$
\mathscr{E}(U)=\left\{e \in \Gamma(U ; E) \text { s.t. } \nabla_{a} e=0, \forall a \in \Gamma(U ; A)\right\}
$$

and

$$
\mathscr{L}(U)=\{l \in \Gamma(U ; L) \text { s.t. }[a, l] \in \Gamma(U ; A), \forall a \in \Gamma(U ; A)\},
$$

where $U$ denotes an arbitrary open subset of $M$.

Lemma 2.1. Given an A-module $E$, there always exists an $L$-connection on $E$ extending the given $A$-connection. Moreover, if $\nabla^{1}$ and $\nabla^{2}$ are two such extensions, then $d^{\nabla^{2}}-d^{\nabla^{1}} \in$ $\Gamma\left(A^{\perp} \otimes\right.$ End $\left.E\right)$, where $A^{\perp}$ denotes the annihilator of $A$ in $L^{*}$. 


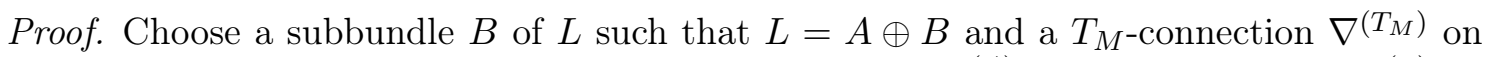
$E$ - this is always possible. Then extend $A$-connection $\nabla^{(A)}$ to an $L$-connection $\nabla^{(L)}$ on $E$ by setting

$$
\nabla_{a+b}^{(L)}=\nabla_{a}^{(A)}+\nabla_{\rho(b)}^{\left(T_{M}\right)}
$$

where $\rho$ denotes the anchor map $L \rightarrow T_{M}$. The difference $l \mapsto \nabla_{l}^{1}-\nabla_{l}^{2}$ of two such extensions $\nabla^{1}$ and $\nabla^{2}$ is a bundle map $L \rightarrow$ End $E$, which vanishes on $A$.

Definition 2.2. An L-connection $\nabla$ on $E$ is said to be A-compatible, if

(a) it extends the given A-action on $E$, and

(b) it satisfies

$$
\nabla_{a} \nabla_{l}-\nabla_{l} \nabla_{a}=\nabla_{[a, l]}, \quad \forall a \in \Gamma(A), l \in \Gamma(L) .
$$

Proposition 2.3. Let $\nabla$ be an L-connection on $E$ extending its A-action. Provided that the sheaf of smooth sections of $E$ is isomorphic to $C_{M}^{\infty} \otimes_{\mathbb{R}} \mathscr{E}$ and the sheaf of smooth sections of $L$ is isomorphic to $C_{M}^{\infty} \otimes_{\mathbb{R}} \mathscr{L}$, the L-connection $\nabla$ is A-compatible if and only if $\nabla \mathscr{L} \mathscr{E} \subset \mathscr{E}$.

Proof. For any $a \in \Gamma(U ; A), l \in \mathscr{L}(U)$ and $e \in \mathscr{E}(U)$, we have $[a, l] \in \Gamma(U ; A), \nabla_{a} e=0$, and $\nabla_{[a, e]}=0$ so that, if $\nabla$ is $A$-compatible, we obtain

$$
\nabla_{a} \nabla_{l} e=\nabla_{a} \nabla_{l} e-\nabla_{l} \nabla_{a} e-\nabla_{[a, l]} e=0 .
$$

Hence $\nabla_{l} e \in \mathscr{E}(U)$. This proves that $\nabla_{\mathscr{L} \mathscr{E}} \subset \mathscr{E}$. Conversely, if $\nabla_{\mathscr{L}} \mathscr{E} \subset \mathscr{E}$, then

$$
\left(\nabla_{a} \nabla_{f \cdot l}-\nabla_{f \cdot l} \nabla_{a}-\nabla_{[a, f \cdot l]}\right)(g \cdot e)=f g \cdot \nabla_{a} \nabla_{l} e=0,
$$

for all $a \in \Gamma(U ; A), f, g \in C_{U}^{\infty}, l \in \mathscr{L}(U)$ and $e \in \mathscr{E}(U)$. Since $\Gamma(U ; E)=C_{U}^{\infty} \otimes_{\mathbb{R}} \mathscr{E}$ and $\Gamma(U ; L)=C_{M}^{\infty} \otimes_{\mathbb{R}} \mathscr{L}$, it follows that $\nabla$ is $A$-compatible.

Remark 2.4. Given a matched pair of Lie algebroids $(A, B)$ and an $A$-module $E$, consider the Lie algebroid $L=A \bowtie B$. An A-compatible $L$-connection on $E$ determines a $B$ connection on $E$ satisfying

$$
\nabla_{a} \nabla_{b} e-\nabla_{b} \nabla_{a} e=\nabla_{[a, b]} e, \quad \forall a \in \Gamma(A), b \in \Gamma(B), e \in \Gamma(E) .
$$

The converse is also true.

2.2.2. Atiyah class: obstruction to compatibility. Assume that $(L, A)$ is a Lie pair, $E$ is an $A$-module, and $\nabla$ is an $L$-connection on $E$ extending its $A$-action. The curvature of $\nabla$ is the bundle map $R^{\nabla}: \wedge^{2} L \rightarrow$ End $E$ defined by

$$
R^{\nabla}\left(l_{1}, l_{2}\right)=\nabla_{l_{1}} \nabla_{l_{2}}-\nabla_{l_{2}} \nabla_{l_{1}}-\nabla_{\left[l_{1}, l_{2}\right]}, \quad \forall l_{1}, l_{2} \in \Gamma(L) .
$$

Since $E$ is an $A$-module, its restriction to $\wedge^{2} A$ vanishes. Hence the curvature induces a section $R_{E}^{\nabla} \in \Gamma\left(A^{*} \otimes A^{\perp} \otimes\right.$ End $\left.E\right)$ or, equivalently, a bundle map $R_{E}^{\nabla}: A \otimes(L / A) \rightarrow$ End $E$ given by

$$
R_{E}^{\nabla}(a ; q(l))=R^{\nabla}(a, l)=\nabla_{a} \nabla_{l}-\nabla_{l} \nabla_{a}-\nabla_{[a, l]}, \quad \forall a \in \Gamma(A), l \in \Gamma(L) .
$$

The $L$-connection $\nabla$ is compatible with the $A$-module structure of $E$ if and only if $R_{E}^{\nabla}=0$.

Theorem 2.5. $\quad$ (a) The section $R_{E}^{\nabla}$ of $A^{*} \otimes A^{\perp} \otimes$ End $E$ is a 1-cocycle for the Lie algebroid $A$ with values in the $A$-module $A^{\perp} \otimes$ End $E$.

(b) The cohomology class $\alpha_{E} \in H^{1}\left(A, A^{\perp} \otimes\right.$ End $\left.E\right)$ of the 1-cocycle $R_{E}^{\nabla}$ does not depend on the choice of $L$-connections extending the A-action.

(c) The Atiyah class $\alpha_{E}$ of $E$ vanishes if and only if there exists an A-compatible $L$-connection on E. 
Proof. We use the symbol $\partial^{A}$ to denote the covariant differential associated to the action of the Lie algebroid $A$ on $A^{\perp} \otimes$ End $E$.

(a) The second Bianchi identity states that $d^{\nabla} R^{\nabla}: \wedge^{3} L \rightarrow$ End $E$ is identically zero. Thus, for any $a_{1}, a_{2} \in \Gamma(A), l \in \Gamma(L)$, we have

$$
\begin{aligned}
0= & \left(d^{\nabla} R^{\nabla}\right)\left(a_{1}, a_{2}, l\right) \\
= & \nabla_{a_{1}}\left(R^{\nabla}\left(a_{2}, l\right)\right)-\nabla_{a_{2}}\left(R^{\nabla}\left(a_{1}, l\right)\right)+\nabla_{l}\left(R^{\nabla}\left(a_{1}, a_{2}\right)\right) \\
& -R^{\nabla}\left(\left[a_{1}, a_{2}\right], l\right)+R^{\nabla}\left(\left[a_{1}, l\right], a_{2}\right)-R^{\nabla}\left(\left[a_{2}, l\right], a_{1}\right) \\
= & \nabla_{a_{1}}\left(R_{E}^{\nabla}\left(a_{2} ; q(l)\right)\right)-\nabla_{a_{2}}\left(R_{E}^{\nabla}\left(a_{1} ; q(l)\right)\right) \\
& -R_{E}^{\nabla}\left(\left[a_{1}, a_{2}\right] ; q(l)\right)-R_{E}^{\nabla}\left(a_{2} ; \nabla_{a_{1}} q(l)\right)+R_{E}^{\nabla}\left(a_{1} ; \nabla_{a_{2}} q(l)\right) \\
= & \left(\nabla_{a_{1}}\left(R_{E}^{\nabla}\left(a_{2} ; q(l)\right)\right)-R_{E}^{\nabla}\left(a_{2} ; \nabla_{a_{1}} q(l)\right)\right) \\
& -\left(\nabla_{a_{2}}\left(R_{E}^{\nabla}\left(a_{1} ; q(l)\right)\right)-R_{E}^{\nabla}\left(a_{1} ; \nabla_{a_{2}} q(l)\right)\right)-R_{E}^{\nabla}\left(\left[a_{1}, a_{2}\right] ; q(l)\right) \\
= & \left(\partial^{A} R_{E}^{\nabla}\right)\left(a_{1}, a_{2} ; q(l)\right) .
\end{aligned}
$$

Therefore $\partial^{A} R_{E}^{\nabla}=0$.

(b) By Lemma 2.1, if $\nabla^{1}$ and $\nabla^{2}$ are two $L$-connections that extend the $A$-action, then $\nabla_{l}^{1}-\nabla_{l}^{2}=\phi(l)$ for some $\phi \in \Gamma\left(A^{\perp} \otimes\right.$ End $\left.E\right)$, and

$$
\begin{aligned}
R_{E}^{\nabla_{1}}(a ; q(l)) \cdot e-R_{E}^{\nabla_{2}}(a ; q(l)) \cdot e \\
\quad=\nabla_{a}\left(\nabla_{l}^{1}-\nabla_{l}^{2}\right) e-\left(\nabla_{l}^{1}-\nabla_{l}^{2}\right) \nabla_{a} e-\left(\nabla_{[a, l]}^{1}-\nabla_{[a, l]}^{2}\right) e \\
\quad=\nabla_{a}(\phi(l) \cdot e)-\phi(l) \cdot\left(\nabla_{a} e\right)-\phi([a, l]) e \\
\quad=\left(\partial^{A} \phi\right)(a ; l) \cdot e .
\end{aligned}
$$

So $R_{E}^{\nabla_{1}}-R_{E}^{\nabla_{2}}=\partial^{A} \phi$.

(c) It is clear that $R_{E}^{\nabla}$ vanishes if and only if $\nabla$ is $A$-compatible. Now, if $R_{E}^{\nabla}=\partial^{A} \phi$ for some $\phi \in \Gamma\left(A^{\perp} \otimes\right.$ End $\left.E\right)$, set $\nabla^{\prime}=\nabla-\phi$. Then $R_{E}^{\nabla^{\prime}}=0$, which implies that $\nabla^{\prime}$ is $A$-compatible.

We call $R_{E}^{\nabla}$ the Atiyah cocycle associated with the $L$-connection $\nabla$ that extends the $A$ module structure of $E$, and its corresponding cohomology class $\alpha_{E} \in H^{1}\left(A, A^{\perp} \otimes\right.$ End $\left.E\right)$ the Atiyah class of the $A$-module $E$.

Remark 2.6. When the Lie pair $(L, A)$ is a matched pair of Lie algebroids, i.e. $L=A \bowtie B$, our definition of Atiyah class is a special case of the Atiyah class of a dDG algebra developed by Calaque and Van den Bergh [12. Hence in the matched pair case, Theorem 2.5 (a)-(b) is a consequence of Lemma 8.2.4 in [12.

Example 2.7. Let $X$ be a complex manifold, and $E$ a holomorphic vector bundle over $X$. Then $A=T_{X}^{0,1}$ and $B=T_{X}^{1,0}$ form a matched pair of Lie algebroids and $L=A \bowtie B$ is isomorphic to $T_{X} \otimes \mathbb{C}$. Moreover $E$ is an $A$-module [30]. It is simple to see that holomorphic $T_{X}$-connections on $E$ are equivalent to $L$-connections on $E$ compatible with the $A$-action (as well as to A-compatible B-connections on $E$ - see Remark 2.4). In this case, the Atiyah cocycle is exactly the Dolbeault 1-cocycle $\mathcal{R}$ defined by Equation (2).

Example 2.8. A holomorphic Lie algebroid $K$ over a complex manifold $X$ yields a matched pair of complex Lie algebroids $\left(T_{X}^{0,1}, K^{1,0}\right)$ [30]. The Atiyah class of the $T_{X}^{0,1}$ module $K^{1,0}$ is the Atiyah class for $K$ studied extensively by Calaque and Van den Bergh in [12].

Example 2.9. In [41], Molino introduced an Atiyah class for connections "transversal to a foliation," which measures the obstruction to their "projectability." Although not phrased 
in the language of Lie algebroids, his construction is a special case of ours. Here $L$ is the tangent bundle $T_{M}, A$ is the tangent bundle to a foliation $\mathcal{F}$ of $M$, and the $A$-module $E$ is a vector bundle on $M$ foliated over $\mathcal{F}$. A transversal connection is an $L$-connection on $E$ which extends the $A$-action. It is said to be projectable precisely if it is $A$-compatible, i.e. if it is preserved by parallel transport along any path tangent to $\mathcal{F}$.

Example 2.10. Let $\mathfrak{g}$ be a Lie subalgebra of a Lie algebra $\mathfrak{d}$. Given a $\mathfrak{g}$-module $E$ (i.e. a Lie algebra morphism $\boldsymbol{A}: \mathfrak{g} \rightarrow$ End $E$ ), and a $\mathfrak{d}$-connection on $E$ extending it (i.e. a linear map $\boldsymbol{L}: \mathfrak{d} \rightarrow$ End $E$ whose restriction to $\mathfrak{g}$ is $\boldsymbol{A})$, the Atiyah class is the element in the Chevalley-Eilenberg cohomology group $H^{1}\left(\mathfrak{g}, \mathfrak{g}^{\perp} \otimes \operatorname{End}(E)\right)$ determined by $\partial^{\mathfrak{g}} \boldsymbol{L}$. (The symbol $\partial^{\mathfrak{g}}$ denotes the Chevalley-Eilenberg coboundary of $\mathfrak{d}^{*} \otimes \operatorname{End}(E)$-valued $\mathfrak{g}$-cochains.) Here $\boldsymbol{L}$ is considered as an element in $\mathfrak{d}^{*} \otimes \operatorname{End}(E)$, which is, in general, not in $\mathfrak{g}^{\perp} \otimes \operatorname{End}(E)$. Hence, in general, $\partial^{\mathfrak{g}} \boldsymbol{L}$ does not vanish in $H^{1}\left(\mathfrak{g}, \mathfrak{g}^{\perp} \otimes \operatorname{End}(E)\right)$.

The following example is due to Calaque-Căldăraru-Tu [10].

Example 2.11. Consider the Lie algebra $\mathfrak{s l}_{2}(\mathbb{C})$ and its standard basis

$$
h=\left(\begin{array}{cc}
1 & 0 \\
0 & -1
\end{array}\right), \quad e=\left(\begin{array}{cc}
0 & 1 \\
0 & 0
\end{array}\right), \quad f=\left(\begin{array}{ll}
0 & 0 \\
1 & 0
\end{array}\right) .
$$

We have

$$
[e, f]=h, \quad[h, e]=2 e, \quad[h, f]=-2 f .
$$

Together, the matrices $h$ and $e$ generate the Lie subalgebra $\mathfrak{g}$ of $2 \times 2$ traceless upper triangular matrices. We identify the quotient $\mathfrak{s l}_{2}(\mathbb{C}) / \mathfrak{g}$ to the nilpotent Lie subalgebra $\mathfrak{n}$ generated by $f$. Note that $\mathfrak{g}$ and $\mathfrak{n}$ form a matched pair of Lie algebras with sum $\mathfrak{g} \oplus \mathfrak{n}=\mathfrak{s l}_{2}(\mathbb{C})$. The bilinear map $\theta: \mathfrak{n} \otimes \mathfrak{n} \rightarrow \mathfrak{n}$ defined by $\theta(f, f)=f$ is a generator of the one-dimensional $\mathfrak{g}$-module $\mathfrak{g}^{\perp} \otimes \operatorname{End}(\mathfrak{n}) \cong \operatorname{Hom}(\mathfrak{n} \otimes \mathfrak{n}, \mathfrak{n})$. The action of $\mathfrak{g}$ on $\operatorname{Hom}(\mathfrak{n} \otimes \mathfrak{n}, \mathfrak{n})$ is given by $h \cdot \theta=2 \theta$ and $e \cdot \theta=0$. One checks that the degree 1 cohomology $H^{1}\left(\mathfrak{g}, \mathfrak{g}^{\perp} \otimes \operatorname{End}(\mathfrak{n})\right)$ is a one-dimensional vector space generated by the Atiyah class $\alpha_{\mathfrak{n}}$ of the $\mathfrak{g}$-module $\mathfrak{n}$.

2.3. Functoriality. Let $M$ and $N$ be smooth manifolds, $f: N \rightarrow M$ a smooth map, $A$ a Lie algebroid over $M$ with anchor $\rho: A \rightarrow T M$, and $E$ a smooth vector bundle over $M$.

Let $f^{*} E$ denote the pullback of $E$ through $f$, i.e. the fibered product of $N$ and $E$ over $M$ :

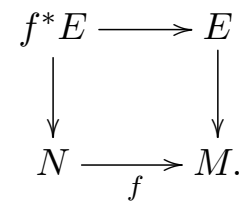

If the anchor $\rho$ and the differential of $f$ are transversal (i.e. $f_{*}(T N)+\rho(A)=\left.T M\right|_{N}$ ), we can consider the fibered product $f^{\star} A$ of $T N$ and $A$ over $T M$ :

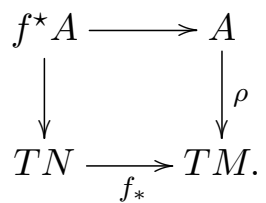

Note that $\rho$ and $f_{*}$ are automatically transversal when $f$ is a surjective submersion or when the Lie algebroid $A$ is transitive. It is clear that $f^{\star} A$ is a vector bundle over $N$. However, note that $f^{\star} A \neq f^{*} A$. The fiber of $f^{\star} A$ over a point $n \in N$ is

$$
\left(f^{\star} A\right)_{n}=\left\{(x, a) \in T_{n} N \oplus A_{f(n)} \mid f_{*}(x)=\rho(a)\right\} .
$$


The Lie algebroid structure on $A$ induces a Lie algebroid structure on $f^{\star} A \rightarrow N$; its anchor is the projection $f^{\star} A \rightarrow T N$ and its bracket is given by

$$
\left[\left(x_{1}, a_{1}\right),\left(x_{2}, a_{2}\right)\right]=\left(\left[x_{1}, x_{2}\right],\left[a_{1}, a_{2}\right]\right),
$$

for any $x_{1}, x_{2} \in \mathfrak{X}(N)$ and $a_{1}, a_{2} \in \Gamma(A)$ such that $f_{*}\left(x_{1}\right)=\rho\left(a_{1}\right)$ and $f_{*}\left(x_{2}\right)=\rho\left(a_{2}\right)$ (see [20] for details).

Proposition 2.12. Let $A$ be a Lie algebroid over $M$ and $f: N \rightarrow M$ a smooth map whose differential $f_{*}: T N \rightarrow T M$ is transversal to the anchor of $A$. Then

(a) If $E$ is a module over $A$, then $f^{*} E$ is a module over $f^{\star} A$.

(b) The map $f$ induces a natural homomorphism

$$
f^{\dagger}: H^{\bullet}(A, E) \rightarrow H^{\bullet}\left(f^{\star} A, f^{*} E\right) .
$$

Proof. The first assertion is easily proved if one thinks of Lie algebroid modules in terms of horizontal lifting. The second assertion follows from a direct verification.

The following proposition is immediate.

Proposition 2.13. If $(L, A)$ is a Lie pair over $M$, and $f: N \rightarrow M$ a smooth map whose differential $f_{*}: T N \rightarrow T M$ is transversal to the anchor of $A$, then $\left(f^{\star} L, f^{\star} A\right)$ is a Lie pair over $N$.

Given a Lie pair $(L, A)$ over a smooth manifold $M$ and a smooth map $f: N \rightarrow M$ whose differential $f_{*}: T N \rightarrow T M$ is transversal to the anchor of $A$ (otherwise $f^{\star} A$ and $f^{\star} L$ could be singular), there is a canonical morphism of vector bundles $f^{\star} L \rightarrow f^{*}(L / A)$ over $N$ :

$$
f^{\star} L \ni\left(x_{n}, a_{f(n)}\right) \mapsto a_{f(n)}+A_{f(n)} \in f^{*}\left(\frac{L}{A}\right),
$$

whose kernel is exactly $f^{\star} A$. In other words, we have an exact sequence of vector bundles

$$
0 \rightarrow f^{\star} A \rightarrow f^{\star} L \rightarrow f^{*}(L / A) \text {. }
$$

Therefore $f^{\star} L / f^{\star} A$ can be seen as a vector subbundle of $f^{*}(L / A)$.

Lemma 2.14. Under the hypothesis of Proposition 2.13, the inclusion

$$
I: \frac{f^{\star} L}{f^{\star} A} \rightarrow f^{*}\left(\frac{L}{A}\right)
$$

intertwines the $f^{\star} A$-module structures of $\left(f^{\star} L\right) /\left(f^{\star} A\right)$ and $f^{*}(L / A)$.

Dualizing it, as a consequence, we obtain the epimorphism of vector bundles

$$
I^{\dagger}: f^{*}\left(A^{\perp}\right) \rightarrow\left(f^{\star} A\right)^{\perp}
$$

which is a morphism of $f^{\star} A$-modules. Note that, when $f$ is a surjective submersion, $I$ is surjective and thus both $I$ and $I^{\dagger}$ are isomorphisms of $f^{\star} A$-modules.

We are now ready to state the main result in this subsection.

Theorem 2.15. Let $(L, A)$ be a Lie pair over $M$, and $f: N \rightarrow M$ a smooth map whose differential $f_{*}: T N \rightarrow T M$ is transversal to the anchor of $A$. Assume that $E$ is an A-module. Then the composition of homomorphisms

$$
H^{1}\left(A, A^{\perp} \otimes \text { End } E\right) \stackrel{f^{\dagger}}{\rightarrow} H^{1}\left(f^{\star} A, f^{*}\left(A^{\perp} \otimes \operatorname{End} E\right)\right) \stackrel{I^{\dagger}}{\rightarrow} H^{1}\left(f^{\star} A,\left(f^{\star} A\right)^{\perp} \otimes \operatorname{End}\left(f^{*} E\right)\right)
$$

maps the Atiyah class of $E$ relative to the Lie pair $(L, A)$ onto the Atiyah class of $f^{*} E$ relative to the Lie pair $\left(f^{\star} L, f^{\star} A\right)$ :

$$
\left(I^{\dagger} \circ f^{\dagger}\right)\left(\alpha_{E}\right)=\alpha_{f^{*} E}
$$


2.4. Scalar Atiyah classes and Todd class. Let $(L, A)$ be a Lie pair. We define the scalar Atiyah classes [3] of an $A$-module $E$ by

$$
c_{k}(E):=\frac{1}{k !}\left(\frac{i}{2 \pi}\right)^{k} \operatorname{tr}\left(\alpha_{E}^{k}\right) \in H^{k}\left(A, \wedge^{k} A^{\perp}\right) .
$$

Here $\alpha_{E}^{k}$ denotes the image of $\alpha_{E} \otimes \cdots \otimes \alpha_{E}$ under the natural map

$$
H^{1}\left(A, A^{\perp} \otimes \text { End } E\right) \times \cdots \times H^{1}\left(A, A^{\perp} \otimes \text { End } E\right) \rightarrow H^{k}\left(A, \wedge^{k} A^{\perp} \otimes \text { End } E\right)
$$

induced by the composition in End $E$ and the wedge product in $\wedge^{\bullet} A^{\perp}$.

Remark 2.16. If $E$ is a holomorphic vector bundle over a compact Kähler manifold $X$, the natural inclusion of $H^{k}\left(X, \Omega^{k}\right)$ into $H^{2 k}(X, \mathbb{C})$ maps the scalar Atiyah classes of $E$ relative to the Lie pair $\left(L=T_{X} \otimes \mathbb{C}, A=T_{X}^{0,1}\right)$ to the Chern classes of $E$.

The Todd class of the $A$-module $E$ relative to the Lie pair $(L, A)$ is the cohomology class

$$
\operatorname{Td}(E)=\operatorname{det}\left(\frac{\alpha_{E}}{1-e^{-\alpha_{E}}}\right) \in H^{\bullet}\left(A, \wedge^{\bullet} A^{\perp}\right) .
$$

The following propositions can be verified directly.

Proposition 2.17. Let $(L, A)$ be a Lie pair and let $E_{1}, E_{2}$ be A-modules. Then

$$
\operatorname{Td}\left(E_{1} \oplus E_{2}\right)=\operatorname{Td} E_{1} \cdot \operatorname{Td} E_{2} .
$$

Proposition 2.18. Under the hypothesis of Theorem 2.15, we have

$$
\begin{aligned}
c_{k}\left(f^{*} E\right) & =\left(I^{\dagger} \circ f^{\dagger}\right)\left(c_{k} E\right) \in H^{k}\left(f^{\star} A, \wedge^{k}\left(f^{\star} A\right)^{\perp}\right) \\
\operatorname{Td}\left(f^{*} E\right) & =\left(I^{\dagger} \circ f^{\dagger}\right)(\operatorname{Td} E) \in H^{\bullet}\left(f^{\star} A, \wedge^{\bullet}\left(f^{\star} A\right)^{\perp}\right)
\end{aligned}
$$

\subsection{Jet short exact sequence.}

2.5.1. The jet bundle $\mathscr{J}_{L / A}^{1} E$. Let $M$ be a smooth manifold, let $L \rightarrow M$ be a Lie algebroid, and let $E \stackrel{\pi}{\rightarrow} M$ be a vector bundle.

An $L$-jet (of order 1 ) on $E\left(\right.$ at $e_{x} \in E$ ) is a linear map $L_{\pi\left(e_{x}\right)} \stackrel{\phi}{\rightarrow} T_{e_{x}} E$ such that the diagram

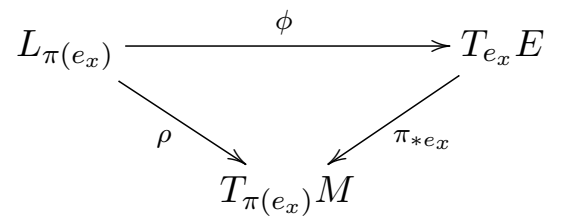

commutes. The jet space $\mathscr{J}_{L}^{1} E$ is the manifold whose points are $L$-jets on $E$. It is a vector bundle over $X$ : the projection $\mathscr{J}_{L}^{1} E \rightarrow M$ maps $\left(L_{\pi\left(e_{x}\right)} \stackrel{\phi}{\rightarrow} T_{e_{x}} E\right)$ to $\pi\left(e_{x}\right)$. It fits into the short exact sequence of vector bundles over $M$ :

$$
0 \longrightarrow L^{*} \otimes E \stackrel{\hat{f}}{\longrightarrow} \mathscr{J}_{L}^{1} E \stackrel{\hat{g}}{\longrightarrow} E \longrightarrow 0 .
$$

The surjection $\hat{g}$ maps $\left(L_{\pi\left(e_{x}\right)} \stackrel{\phi}{\rightarrow} T_{e_{x}} E\right)$ to $e_{x}$, while the injection $\hat{f}$ maps $\left(L_{x} \stackrel{\psi}{\rightarrow} E_{x}\right)$ to $\left(L_{x} \stackrel{\rho \oplus \psi}{\longrightarrow} T_{x} M \oplus E_{x} \cong T_{0_{x}} E\right)$.

The following result is straightforward.

Proposition 2.19. A splitting $s: E \rightarrow \mathscr{J}_{L}^{1} E$ of the short exact sequence of vector bundles (4) determines a (linear) L-connection on $E$. The converse is also true. 
In general, there is no canonical choice of splitting for (4). However, the induced short exact sequence

$$
0 \longrightarrow \Gamma\left(L^{*} \otimes E\right) \stackrel{\hat{f}_{\sharp}}{\longrightarrow} \Gamma\left(\mathscr{J}_{L}^{1} E\right) \stackrel{\hat{g}_{\sharp}}{\longrightarrow} \Gamma(E) \longrightarrow 0
$$

at the level of spaces of smooth sections splits canonically: if $e$ is a section of $E$, then $\sigma e:=e_{*} \circ \rho$ is a section of $\mathscr{J}_{L}^{1} E$ such that $\hat{g}_{\sharp}(\sigma e)=e$.

We note that the covariant differential $d^{\nabla}: \Gamma(E) \rightarrow \Gamma\left(L^{*} \otimes E\right)$ associated to a splitting $s: E \rightarrow \mathscr{J}_{L}^{1} E$ of the short exact sequence (4) is given by

$$
\hat{f}_{\sharp}\left(d^{\nabla} e\right)=\sigma e-s_{\sharp}(e), \quad \forall e \in \Gamma(E) .
$$

Now assume $A$ is a Lie subalgebroid of $L$ and $E$ is an $A$-module. The symbol $h$ will denote the horizontal lifting associated to the $A$-action on $E$.

An $h$-extending $L$-jet (of order 1 ) on $E$ is a linear map $L_{\pi\left(e_{x}\right)} \stackrel{\phi}{\rightarrow} T_{e_{x}} E$ such that the diagram

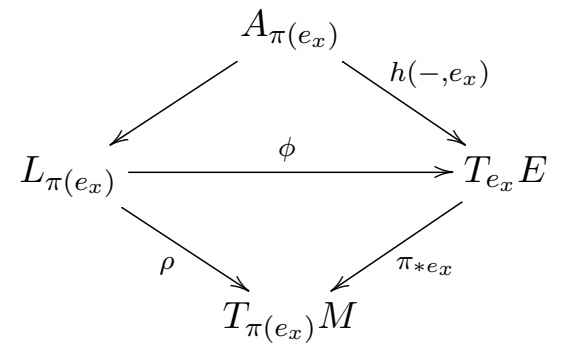

commutes. The jet space $\mathscr{J}_{L / A}^{1} E$ is the manifold whose points are $h$-extending $L$-jets on $E$. It is a vector bundle over $M$ : the projection $\mathscr{J}_{L / A}^{1} E \rightarrow M$ maps $\left(L_{\pi\left(e_{x}\right)} \stackrel{\phi}{\rightarrow} T_{e_{x}} E\right)$ to $\pi\left(e_{x}\right)$.

Example 2.20. When $E$ is a holomorphic vector bundle over a complex manifold $X$, $A=T_{X}^{0,1}$ and $L=T_{X} \otimes \mathbb{C}$, the jet bundle $\mathscr{J}_{L / A}^{1} E$ is simply the bundle of jets (of order 1 ) of holomorphic sections of $E$.

Consider the surjective morphism of vector bundles $\breve{g}: \mathscr{J}_{L / A}^{1} E \rightarrow E$, which maps $\left(L_{\pi\left(e_{x}\right)} \stackrel{\phi}{\rightarrow}\right.$ $\left.T_{e_{x}} E\right)$ to $e_{x}$. Since $T_{0_{x}} E$ is canonically isomorphic to $T_{x} M \oplus E_{x}$, the kernel of $\breve{g}$ can be identified naturally with the subbundle $K$ of $L^{*} \otimes E \rightarrow M$ consisting of all linear maps $\left(L_{x} \stackrel{\psi}{\rightarrow} E_{x}\right)$ which satisfy

$$
h\left(a_{x}, 0_{x}\right)=\rho\left(a_{x}\right)+\psi\left(a_{x}\right), \quad \forall x \in M, a_{x} \in A_{x} .
$$

Since the $A$-connection $h$ on $E$ is linear, $h\left(a_{x}, 0_{x}\right)$ must be the image of $\rho\left(a_{x}\right)$ under the differential of the zero section $M \stackrel{0}{\rightarrow} E$. Therefore, a linear map $\left(L_{x} \stackrel{\psi}{\rightarrow} E_{x}\right)$ is an element of $K$ if and only if $\psi\left(a_{x}\right)=0$ for all $a_{x} \in A$, so that $K \cong A^{\perp} \otimes E$. Hence we obtain the short exact sequence of vector bundles

$$
0 \longrightarrow A^{\perp} \otimes E \stackrel{\breve{f}}{\longrightarrow} \mathscr{J}_{L / A}^{1} E \stackrel{\breve{g}}{\longrightarrow} E \longrightarrow 0
$$

where the injection $\breve{f}$ maps $\left(L_{x} \stackrel{\psi}{\rightarrow} E_{x}\right)$ to the jet

$$
L_{x} \rightarrow T_{0_{x}} E \cong T_{x} M \oplus E_{x} \quad l_{x} \mapsto \rho\left(l_{x}\right)+\psi\left(l_{x}\right) .
$$

In general, there is no canonical choice of splitting for (6). 
Proposition 2.21. A splitting $s: E \rightarrow \mathscr{J}_{L / A}^{1} E$ of the short exact sequence of vector bundles (6) determines a (linear) L-connection on $E$ extending the $A$-action $h$. The converse is also true.

Obviously, we have the commutative diagram with exact rows

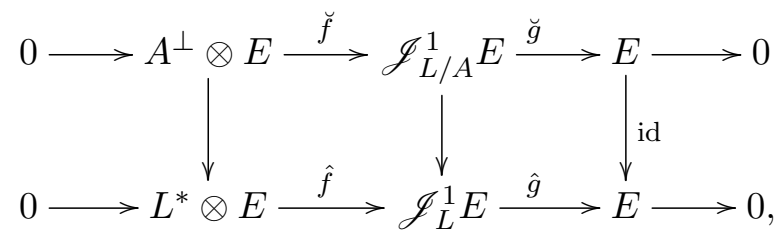

all of whose vertical arrows denote inclusions.

\subsubsection{An equivalent description of the jet bundle.}

Proposition 2.22. An L-jet (of order 1) on E extending the A-action $\nabla$ is a pair $\left(D_{x}, e_{x}\right)$ consisting of a linear map $D_{x}: \Gamma\left(E^{*}\right) \rightarrow L_{x}^{*}$ and a point $e_{x}$ in the fiber of $E$ over $x \in M$, satisfying

$$
\begin{gathered}
\left\langle D_{x}(\varepsilon), a_{x}\right\rangle=\left\langle\nabla_{a_{x}} \varepsilon, e_{x}\right\rangle \quad\left(\text { or equivalently } \quad D_{x}(\varepsilon)=\left\langle d^{\nabla} \varepsilon, e_{x}\right\rangle\right) ; \\
D_{x}(f \varepsilon)=f(x) \cdot D_{x}(\varepsilon)+\left\langle\varepsilon_{x}, e_{x}\right\rangle \cdot \rho^{*}(d f),
\end{gathered}
$$

for all $a_{x} \in A_{x}, \varepsilon \in \Gamma\left(E^{*}\right)$, and $f \in C^{\infty}(M)$.

Proof. Given such a pair $\left(D_{x}, e_{x}\right)$, each $l_{x} \in L_{x}$ determines uniquely a tangent vector $\tau_{x} \in T_{e_{x}} E$ through the relations

$$
\tau_{x}\left(\pi^{*} f\right)=\rho\left(l_{x}\right) f=\left\langle\rho^{*}(d f), l_{x}\right\rangle \quad \text { and } \quad \tau_{x}\left(f_{\varepsilon}\right)=\left\langle D_{x}(\varepsilon), l_{x}\right\rangle,
$$

where $\varepsilon \in \Gamma\left(E^{*}\right), f \in C^{\infty}(M)$, and $f_{\varepsilon} \in C^{\infty}(E)$ is the fiberwise linear function associated to $\varepsilon$ :

$$
f_{\varepsilon}\left(e_{x}\right)=\left\langle\varepsilon_{x}, e_{x}\right\rangle
$$

Let $\phi_{D}: L_{x} \rightarrow T_{e_{x}} E$ be the map $l_{x} \mapsto \tau_{x}$. Clearly, $\phi_{D}$ is linear and satisfies $\pi_{*} \circ \phi_{D}=\rho$. Moreover, $\phi_{D}$ is an extension of the $A$-action since

$$
\left(\phi_{D}\left(a_{x}\right)\right)\left(f_{\varepsilon}\right)=\left\langle D_{x}(\varepsilon), a_{x}\right\rangle=\left\langle\nabla_{a_{x}} \varepsilon, e_{x}\right\rangle=h\left(a_{x}, e_{x}\right)\left(f_{\varepsilon}\right) .
$$

Here we have made use of Equation (1). Hence $\phi_{D} \in\left(\mathscr{J}_{L / A}^{1} E\right)_{x}$ and $\breve{g}\left(\phi_{D}\right)=e_{x}$. Conversely, given an element $\phi: L_{x} \rightarrow T_{e_{x}} E$ of $\left(\mathscr{J}_{L / A}^{1} E\right)_{x}$ that projects to $e_{x}$ under $\breve{g}$, we can define a linear map $D_{x}^{\phi}: \Gamma\left(E^{*}\right) \rightarrow L_{x}^{*}$ by the relation

$$
\left\langle D_{x}^{\phi}(\varepsilon), l_{x}\right\rangle=\left(\phi\left(l_{x}\right)\right)\left(f_{\varepsilon}\right) .
$$

It is straightforward to check that $\left(D_{x}^{\phi}, e_{x}\right)$ satisfies (7) and (8).

Remark 2.23. The surjection $\breve{g}: \mathscr{J}_{L / A}^{1} E \rightarrow E$ in (6) maps the 1-jet $\left(D_{x}, e_{x}\right)$ to $e_{x}$. The injection $\breve{f}$ in (6) maps $\psi \in\left(A^{\perp} \otimes E\right)_{x}$ to $\left(\psi_{x}^{\dagger}, 0_{x}\right) \in\left(\mathscr{J}_{L / A}^{1} E\right)_{x}$, where $0_{x}$ is the zero vector of $E_{x}$ and $\psi_{x}^{\dagger}: \Gamma\left(E^{*}\right) \rightarrow L_{x}^{*}$ is the linear map defined by

$$
\left\langle\psi_{x}^{\dagger}(\varepsilon), l_{x}\right\rangle=\left\langle\varepsilon_{x}, \psi\left(l_{x}\right)\right\rangle, \quad \forall l_{x} \in L_{x}, \varepsilon \in \Gamma\left(E^{*}\right) .
$$

Here $\psi$ is considered as a linear map $L_{x} \rightarrow E_{x}$ whose kernel contains $A_{x}$. 
2.5.3. The jet bundle as an A-module. The jet bundle $\mathscr{J}_{L / A}^{1} E$ can be naturally endowed with an $A$-action.

In the language of Proposition 2.22, a section of $\mathscr{J}_{L / A}^{1} E \rightarrow M$ consists of a section $e$ of $E \rightarrow M$ and an $\mathbb{R}$-linear map $D: \Gamma\left(E^{*}\right) \rightarrow \Gamma\left(L^{*}\right)$ satisfying

$$
\begin{gathered}
\langle D(\varepsilon), a\rangle=\left\langle\nabla_{a} \varepsilon, e\right\rangle ; \\
D(f \varepsilon)=f \cdot D(\varepsilon)+\langle\varepsilon, e\rangle \cdot \rho^{*}(d f),
\end{gathered}
$$

for all $f \in C^{\infty}(M), \varepsilon \in \Gamma\left(E^{*}\right)$, and $a \in \Gamma(A)$.

Proposition 2.24. (a) The jet bundle $\mathscr{J}_{L / A}^{1} E$ is a module over A; the covariant derivative

is given by

$$
\Gamma(A) \times \Gamma\left(\mathscr{J}_{L / A}^{1} E\right) \rightarrow \Gamma\left(\mathscr{J}_{L / A}^{1} E\right)
$$

$$
\nabla_{a}(D, e)=\left(\nabla_{a} D, \nabla_{a} e\right)
$$

where the $\mathbb{R}$-linear map $\nabla_{a} D: \Gamma\left(E^{*}\right) \rightarrow \Gamma\left(L^{*}\right)$ is defined as follows:

$$
\left\langle\left(\nabla_{a} D\right)(\varepsilon), l\right\rangle=\rho(a)\langle D(\varepsilon), l\rangle-\left\langle D\left(\nabla_{a} \varepsilon\right), l\right\rangle-\langle D(\varepsilon),[a, l]\rangle .
$$

(b) Diagram (6) is a short exact sequence of A-modules.

Proof. (a) Checking that (10) determines a connection is straightforward. The flatness of the $A$-connection on $\mathscr{J}_{L / A}^{1} E$ is a consequence of the flatness of the $A$-connection on $E$.

(b) By definition, $\breve{g}$ is a morphism of $A$-modules. Let us check that $\breve{f}$ is also a morphism of $A$-modules. For any $\psi \in \Gamma\left(A^{\perp} \otimes E\right)$, we have

$$
\begin{aligned}
& \left\langle\left(\nabla_{a} \psi^{\dagger}\right)(\varepsilon), l\right\rangle=\rho(a)\left\langle\psi^{\dagger}(\varepsilon), l\right\rangle-\left\langle\psi^{\dagger}\left(\nabla_{a} \varepsilon\right), l\right\rangle-\left\langle\psi^{\dagger}(\varepsilon),[a, l]\right\rangle \\
& \quad=\rho(a)\langle\psi(l), \varepsilon\rangle-\left\langle\psi(l), \nabla_{a} \varepsilon\right\rangle-\langle\psi([a, l]), \varepsilon\rangle=\left\langle\left(\nabla_{a} \psi\right)(l), \varepsilon\right\rangle=\left\langle\left(\nabla_{a} \psi\right)^{\dagger}(\varepsilon), l\right\rangle .
\end{aligned}
$$

Here the map $\psi^{\dagger}$ is defined by Equation (9).

2.5.4. Alternative description of the A-action on $\mathscr{J}_{L / A}^{1} E$. In this section, $B$ denotes the quotient $L / A$ of the Lie pair $(L, A)$.

The proof of the following lemma is a tedious computation, which we omit.

Lemma 2.25. The splitting $\sigma: \Gamma(E) \rightarrow \Gamma\left(\mathscr{J}_{L}^{1} E\right)$ of the short exact sequence (5) is not $C^{\infty}(M)$-linear. For every $e \in \Gamma(E)$ and $f \in C^{\infty}(M)$, we have

$$
\sigma(f \cdot e)-f \cdot \sigma e=\hat{f}_{\sharp}\left(\rho^{*}(d f) \otimes e\right) .
$$

In general, $\sigma e$ need not be a section of $\mathscr{J}_{L / A}^{1} E$. Nevertheless, fixing a splitting of the short exact sequence of vector bundles

$$
0 \longrightarrow A \stackrel{i}{\longrightarrow} L \stackrel{q}{\longrightarrow} B \longrightarrow 0 \text {, }
$$

i.e. a pair of maps $j: B \rightarrow L$ and $p: L \rightarrow A$ such that $q \circ j=\mathrm{id}_{B}, p \circ i=\mathrm{id}_{A}$ and $i \circ p+j \circ q=\operatorname{id}_{L}$ :

$$
0 \underset{\gtrless}{\rightleftarrows} \stackrel{i}{\underset{p}{\rightleftarrows}} L \stackrel{q}{\underset{j}{\rightleftarrows}} B \underset{\rightleftarrows}{\rightleftarrows},
$$

naturally determines a splitting $\varsigma: \Gamma(E) \rightarrow \Gamma\left(\mathscr{J}_{L / A}^{1} E\right)$ of the short exact sequence of spaces of smooth sections

$$
0 \longrightarrow \Gamma\left(A^{\perp} \otimes E\right) \stackrel{\breve{f}_{\sharp}}{\longrightarrow} \Gamma\left(\mathscr{J}_{L / A}^{1} E\right) \stackrel{\breve{g}_{\sharp}}{\longrightarrow} \Gamma(E) \longrightarrow 0
$$


induced by (6). The image of $x \in M$ under the section $\varsigma e$ of $\mathscr{J}_{L / A}^{1} E$ associated to a section $e$ of $E$ by the splitting $\varsigma$ is the 1-jet

$$
L_{x} \ni l_{x} \stackrel{(\varsigma e)_{x}}{\longrightarrow} h\left(p\left(l_{x}\right), e_{x}\right)+e_{* x}\left(\rho \circ j \circ q\left(l_{x}\right)\right) \in T_{e_{x}} E .
$$

It is not difficult to see that $\breve{g}_{\sharp}(\varsigma e)=e$.

The proof of the following lemma is a tedious computation, which we omit.

Lemma 2.26. The splitting $\varsigma: \Gamma(E) \rightarrow \Gamma\left(\mathscr{J}_{L / A}^{1} E\right)$ is not $C^{\infty}(M)$-linear. For every $e \in \Gamma(E)$ and $f \in C^{\infty}(M)$, we have

$$
\varsigma(f \cdot e)-f \cdot \varsigma e=\breve{f}_{\sharp}((l \mapsto \rho(j \circ q(l)) f) \otimes e) .
$$

Since both $E$ and $A^{\perp}$ are modules over $A$, so is $A^{\perp} \otimes E$ :

$$
\left\langle\nabla_{a}(\lambda \otimes e), l\right\rangle=\rho(a)(\lambda(l)) \cdot e-\lambda([a, l]) \cdot e+\lambda(l) \cdot \nabla_{a} e,
$$

where $a \in \Gamma(A), \lambda \in \Gamma\left(A^{\perp}\right), e \in \Gamma(E)$, and $l \in \Gamma(L)$.

Remark 2.27. Since $\Gamma\left(\mathscr{J}_{L / A}^{1} E\right)$ is the direct sum of $\breve{f}_{\sharp}\left(\Gamma\left(A^{\perp} \otimes E\right)\right)$ and $\varsigma(\Gamma(E))$, to define an $A$-module structure on the jet bundle $\mathscr{J}_{L / A}^{1} E$, it suffices to define the $A$-action on $\breve{f}_{\sharp}\left(\Gamma\left(A^{\perp} \otimes E\right)\right)$ and $\varsigma(\Gamma(E))$. Naively, one might expect to define such an $A$-action by setting

$$
\begin{aligned}
\nabla_{a}\left(\breve{f}_{\sharp}(\lambda \otimes e)\right) & =\breve{f}_{\sharp}\left(\nabla_{a}(\lambda \otimes e)\right) ; \\
\nabla_{a}(\varsigma e) & =\varsigma\left(\nabla_{a} e\right) .
\end{aligned}
$$

However, according to a tedious computation, we have

$$
\varsigma\left(\nabla_{f \cdot a} e\right)-\nabla_{f \cdot a}(\varsigma e)=f \cdot\left(\varsigma\left(\nabla_{a} e\right)-\nabla_{a}(\varsigma e)\right)+\breve{f}_{\sharp}\left((l \mapsto \rho(j \circ q(l)) f) \otimes \nabla_{a} e\right)
$$

and

$$
\varsigma\left(\nabla_{a}(f \cdot e)\right)-\nabla_{a}(\varsigma(f \cdot e))=f \cdot\left(\varsigma\left(\nabla_{a} e\right)-\nabla_{a}(\varsigma e)\right)+\breve{f}_{\sharp}((l \mapsto \rho(i \circ p[j \circ q(l), a]) f) \otimes e),
$$

for any $a \in \Gamma(A), e \in \Gamma(E)$, and $f \in C^{\infty}(M)$. This means that Equation (12) must be modified.

To modify Equation (12), let us introduce some new notations. Given $a \in \Gamma(A)$ and $e \in \Gamma(E)$, define $\Theta(a, e) \in \Gamma\left(A^{\perp} \otimes E\right)$ by the relation

$$
\langle\Theta(a, e), l\rangle=\nabla_{i \circ p[j \circ q(l), a]} e, \quad \forall l \in \Gamma(L) .
$$

The proof of the following Lemma is a tedious computation, which we omit.

Lemma 2.28. For any $f \in C^{\infty}(M)$,

$$
\begin{gathered}
\Theta(f \cdot a, e)-f \cdot \Theta(a, e)=(l \mapsto \rho(j \circ q(l)) f) \otimes \nabla_{a} e ; \\
\Theta(a, f \cdot e)-f \cdot \Theta(a, e)=(l \mapsto \rho(i \circ p[j \circ q(l), a]) f) \otimes e .
\end{gathered}
$$

This suggests the following proposition.

Proposition 2.29. (a) The jet bundle $\mathscr{J}_{L / A}^{1} E \rightarrow M$ is an A-module: the flat $A$ connection on $\mathscr{J}_{L / A}^{1} E$ is given by

$$
\begin{aligned}
& \nabla_{a}\left(\breve{f}_{\sharp}(\lambda \otimes e)\right)=\breve{f}_{\sharp}\left(\nabla_{a}(\lambda \otimes e)\right) \\
& \nabla_{a}(\varsigma e)=\varsigma\left(\nabla_{a} e\right)-\breve{f}_{\sharp}(\Theta(a, e)),
\end{aligned}
$$

for all $a \in \Gamma(A), \lambda \in \Gamma\left(A^{\perp}\right)$, and $e \in \Gamma(E)$. 
(b) Diagram (6) is a short exact sequence of A-modules.

Sketch of proof. (a) It follows from Lemmas 2.26 and 2.28 that what we have defined is indeed a covariant derivative. Flatness follows from the Jacobi identity of the Lie algebroid $L$, and the flatness of the $A$-connections on $E$ and $A^{\perp} \otimes E$.

(b) It suffices to check that $\breve{g}$ is a morphism of $A$-modules. We have

$$
\breve{g}_{\sharp}\left(\nabla_{a}(\varsigma e)\right)=\breve{g}_{\sharp}\left(\varsigma\left(\nabla_{a} e\right)\right)-\breve{g}_{\sharp} \breve{f}_{\sharp}(\Theta(a, e))=\nabla_{a} e=\nabla_{a}\left(\breve{g}_{\sharp}(\varsigma e)\right) .
$$

Remark 2.30. Observe that, for a matched pair $L=A \bowtie B, \Theta(a, e) \in \Gamma\left(B^{*} \otimes E\right)$ is given by the simple formula:

$$
\langle\Theta(a, e), b\rangle=\nabla_{\Delta_{b} a} e .
$$

Proposition 2.31. The A-actions on $\mathscr{J}_{L / A}^{1} E$ defined in Propositions 2.24 and 2.29 are identical.

Proof. The $\mathbb{R}$-linear map $D^{\varsigma e}: \Gamma\left(E^{*}\right) \rightarrow \Gamma\left(L^{*}\right)$ determined by the section se of $\mathscr{J}_{L / A}^{1} E \rightarrow$ $M$ (as explained in the proof of Proposition 2.22) satisfies

$$
\begin{gathered}
\left\langle D^{\varsigma e}(\varepsilon), a\right\rangle=\left\langle\nabla_{a} \varepsilon, e\right\rangle ; \\
\left\langle D^{\varsigma e}(\varepsilon), j(b)\right\rangle=(\varsigma e(j(b)))\left(f_{\varepsilon}\right)=\left(e_{*} \circ \rho(j(b))\right)\left(f_{\varepsilon}\right)=\rho(j(b))\langle\varepsilon, e\rangle,
\end{gathered}
$$

for all $e \in \Gamma(E), \varepsilon \in \Gamma\left(E^{*}\right), a \in \Gamma(A)$, and $b \in \Gamma(B)$. The image of $D^{\text {se }}$ under the action of $a \in \Gamma(A)$ is the $\mathbb{R}$-linear map $\nabla_{a} D^{\varsigma e}: \Gamma\left(E^{*}\right) \rightarrow \Gamma\left(L^{*}\right)$ defined by 10 . We have

$$
\begin{aligned}
& \left\langle\left(\nabla_{a^{\prime}} D^{\varsigma e}\right)(\varepsilon), a\right\rangle \\
& \quad=\rho\left(a^{\prime}\right)\left\langle D^{\varsigma e}(\varepsilon), a\right\rangle-\left\langle D^{\varsigma e}\left(\nabla_{a^{\prime}} \varepsilon\right), a\right\rangle-\left\langle D^{\varsigma e}(\varepsilon),\left[a^{\prime}, a\right]\right\rangle \\
& \quad=\rho\left(a^{\prime}\right)\left\langle\nabla_{a} \varepsilon, e\right\rangle-\left\langle\nabla_{a} \nabla_{a^{\prime}} \varepsilon, e\right\rangle-\left\langle\nabla_{\left[a^{\prime}, a\right]} \varepsilon, e\right\rangle \\
& =\rho\left(a^{\prime}\right)\left\langle\nabla_{a} \varepsilon, e\right\rangle-\left\langle\nabla_{a^{\prime}} \nabla_{a} \varepsilon, e\right\rangle \\
& =\left\langle\nabla_{a} \varepsilon, \nabla_{a^{\prime}} e\right\rangle \\
& =\left\langle D^{\varsigma\left(\nabla_{a^{\prime}} e\right)}(\varepsilon), a\right\rangle
\end{aligned}
$$

and

$$
\begin{aligned}
&\left\langle\left(\nabla_{a^{\prime}} D^{\varsigma e}\right)(\varepsilon), j(b)\right\rangle \\
&= \rho\left(a^{\prime}\right)\left\langle D^{\varsigma e}(\varepsilon), j(b)\right\rangle-\left\langle D^{\varsigma e}\left(\nabla_{a^{\prime}} \varepsilon\right), j(b)\right\rangle-\left\langle D^{\varsigma e}(\varepsilon),\left[a^{\prime}, j(b)\right]\right\rangle \\
&= \rho\left(a^{\prime}\right) \rho(j(b))\langle\varepsilon, e\rangle-\rho(j(b))\left\langle\nabla_{a^{\prime}} \varepsilon, e\right\rangle \\
&-\left\langle D^{\varsigma e}(\varepsilon),-p\left[j(b), a^{\prime}\right]+j\left(\nabla_{a^{\prime}} b\right)\right\rangle \\
&= \rho\left(a^{\prime}\right) \rho(j(b))\langle\varepsilon, e\rangle-\rho(j(b)) \rho\left(a^{\prime}\right)\langle\varepsilon, e\rangle+\rho(j(b))\left\langle\varepsilon, \nabla_{a^{\prime}} e\right\rangle \\
&+\left\langle\nabla_{p\left[j(b), a^{\prime}\right]} \varepsilon, e\right\rangle-\rho\left(j\left(\nabla_{a^{\prime}} b\right)\right)\langle\varepsilon, e\rangle \\
&= \rho\left(\left[a^{\prime}, j(b)\right]\right)\langle\varepsilon, e\rangle+\rho(j(b))\left\langle\varepsilon, \nabla_{a^{\prime}} e\right\rangle+\rho\left(p\left[j(b), a^{\prime}\right]\right)\langle\varepsilon, e\rangle \\
&-\left\langle\varepsilon, \nabla_{p\left[j(b), a^{\prime}\right]} e\right\rangle-\rho\left(j\left(\nabla_{a^{\prime}} b\right)\right)\langle\varepsilon, e\rangle \\
&= \rho(j(b))\left\langle\varepsilon, \nabla_{a^{\prime}} e\right\rangle-\left\langle\varepsilon, \nabla_{p\left[j(b), a^{\prime}\right]} e\right\rangle \\
&=\left\langle D^{\varsigma\left(\nabla_{a^{\prime}} e\right)}(\varepsilon), j(b)\right\rangle-\left\langle\left(\Theta\left(a^{\prime}, e\right)\right)^{\dagger}(\varepsilon), j(b)\right\rangle .
\end{aligned}
$$

Therefore, we obtain

$$
\nabla_{a^{\prime}} D^{\varsigma e}=D^{\varsigma\left(\nabla_{a^{\prime}} e\right)}-\left(\Theta\left(a^{\prime}, e\right)\right)^{\dagger},
$$

which is equivalent to Equation (14). 
2.5.5. The abelian category $\mathcal{A}$. It is a classical result that the space $\Gamma(A)$ of smooth sections of a Lie algebroid $A$ over a smooth manifold $M$ is a Lie-Rinehart algebra over the commutative ring $C^{\infty}(M)$ [21, 44]. We denote the abelian category of modules over this Lie-Rinehart algebra by the symbol $\mathcal{A}$. Alternatively, $\mathcal{A}$ can be seen as the category of left modules over the universal enveloping algebra $\mathcal{U}(A)$ [55] of the Lie algebroid $A$. In particular, the space of smooth sections of an $A$-module, i.e. a vector bundle over $M$ endowed with an $A$-action, is an object in $\mathcal{A}$. By $D^{+}(\mathcal{A})$, we denote the bounded below derived category of $\mathcal{A}$, which is a symmetric monoidal category [22]. The interchange isomorphism $\tau: X \otimes Y \rightarrow Y \otimes X$ of a pair of objects $X$ and $Y$ of $D^{+}(\mathcal{A})$ is given by

$$
\tau(x \otimes y)=(-1)^{|x||y|} y \otimes x .
$$

2.5.6. Extension class of the jet sequence. A short exact sequence of $A$-modules

$$
0 \longrightarrow P \stackrel{\alpha}{\longrightarrow} Q \stackrel{\beta}{\longrightarrow} R \longrightarrow 0
$$

determines an extension class in the group $\operatorname{Ext}_{\mathcal{A}}^{1}(R, P)$, which is naturally isomorphic to the Lie algebroid cohomology group $H^{1}\left(A, R^{*} \otimes P\right)[21$.

Indeed, given a homomorphism of vector bundles $s: R \rightarrow Q$ such that $\beta \circ s=\mathrm{id}_{R}$, we have

$$
s_{\sharp}\left(\nabla_{a} r\right)-\nabla_{a}\left(s_{\sharp}(r)\right) \in \operatorname{ker} \beta, \quad \forall a \in \Gamma(A), r \in \Gamma(R)
$$

so that the equation

$$
s_{\sharp}\left(\nabla_{a} r\right)-\nabla_{a}\left(s_{\sharp}(r)\right)=\alpha_{\sharp}\left(\xi_{s}(a) \cdot r\right)
$$

defines a vector bundle map $\xi_{s}: A \rightarrow \operatorname{Hom}(R, P)$. Rewriting (17) as

$$
\left(\operatorname{id}_{R^{*}} \otimes \alpha\right) \circ \xi_{s}=\partial^{A} s
$$

and recalling that $\alpha: P \rightarrow Q$ is a morphism of $A$-modules, we immediately see that

$$
\left(\operatorname{id}_{R^{*}} \otimes \alpha\right) \circ \partial^{A} \xi_{s}=\partial^{A}\left(\left(\operatorname{id}_{R^{*}} \otimes \alpha\right) \circ \xi_{s}\right)=\partial^{A}\left(\partial^{A} s\right)=0 .
$$

Therefore $\partial^{A} \xi_{s}=0$, i.e. $\xi_{s}$ is a 1-cocycle for the Lie algebroid $A$ with values in the $A$-module $R^{*} \otimes P$. It follows from Equation $[18)$ that the cohomology class $\left[\xi_{s}\right] \in H^{1}\left(A, R^{*} \otimes P\right)$ of the 1-cocycle $\xi_{s}$ defined by (17) is independent of the choice of the section $s: R \rightarrow Q$. In fact, $\left[\xi_{s}\right]$ is the extension class in $\operatorname{Ext}_{\mathcal{A}}^{1}(R, P) \cong H^{1}\left(A, R^{*} \otimes P\right)$ of the short exact sequence of $A$-modules (16).

Proposition 2.32. Given a Lie pair $(L, A)$ and an $A$-module $E$, let $\nabla$ denote the $L$ connection on $E$ determined by a section $s: E \rightarrow \mathscr{J}_{L / A}^{1} E$ of the short exact sequence (6). When considered as sections of $A^{*} \otimes A^{\perp} \otimes \operatorname{End} E$, the bundle maps $\xi_{s}: A \rightarrow \operatorname{Hom}\left(E, A^{\perp} \otimes E\right)$ and $R_{E}^{\nabla}: A \otimes(L / A) \rightarrow$ End $E$ (respectively defined by (17) and (3)) are one and the same.

Proof. Define $\breve{d}^{\nabla}: \Gamma(E) \rightarrow \Gamma\left(A^{\perp} \otimes E\right)$ by

$$
\breve{f}_{\sharp}\left(\breve{d}^{\nabla} e\right)=\varsigma e-s_{\sharp}(e), \quad \forall e \in \Gamma(E) .
$$

Then, for all $b \in B$, we have $\langle\breve{d} \nabla e, j(b)\rangle=\nabla_{j(b)} e$.

For all $a \in \Gamma(A)$, and $e \in \Gamma(E)$, we have

$$
\begin{array}{rlrl}
\breve{f_{\sharp}}\left(\xi_{s}(a) \cdot e\right) & \\
& =s_{\sharp}\left(\nabla_{a} e\right)-\nabla_{a}\left(s_{\sharp} e\right) & & \text { by } 17), \\
& =\left(\varsigma\left(\nabla_{a} e\right)-\breve{f}_{\sharp} \breve{d}^{\nabla}\left(\nabla_{a} e\right)\right)-\left(\nabla_{a}(\varsigma e)-\nabla_{a} \breve{f}_{\sharp}\left(\breve{d}^{\nabla} e\right)\right) & & \text { by 19), } \\
& =\breve{f}_{\sharp}\left(\Theta(a, e)+\nabla_{a}\left(\breve{d}^{\nabla} e\right)-\breve{d}^{\nabla}\left(\nabla_{a} e\right)\right) & & \text { by } 13 \text { and (14). }
\end{array}
$$


Hence, for all $b \in \Gamma(B)$, we get

$$
\begin{aligned}
\left\langle\xi_{s}(a)\right. & \cdot e, j(b)\rangle \\
& =\left\langle\Theta(a, e)+\nabla_{a}\left(\breve{d}^{\nabla} e\right)-\breve{d}^{\nabla}\left(\nabla_{a} e\right), j(b)\right\rangle \\
& =\nabla_{p[j(b), a]} e+\nabla_{a}\langle\breve{d} e, j(b)\rangle-\left\langle\breve{d}^{\nabla} e, j\left(\nabla_{a} b\right)\right\rangle-\left\langle\breve{d}^{\nabla}\left(\nabla_{a} e\right), j(b)\right\rangle \\
& =\nabla_{p[j(b), a]} e+\nabla_{a} \nabla_{j(b)} e-\nabla_{j\left(\nabla_{a} b\right)} e-\nabla_{j(b)} \nabla_{a} e \\
& =\nabla_{a} \nabla_{j(b)} e-\nabla_{j(b)} \nabla_{a} e-\nabla_{j\left(\nabla_{a} b\right)+p[a, j(b)]} e \\
& =\nabla_{a} \nabla_{j(b)} e-\nabla_{j(b)} \nabla_{a} e-\nabla_{[a, j(b)]} e \\
& =R^{\nabla}(a, j(b)) e \\
& =R_{E}^{\nabla}(a ; b) \cdot e .
\end{aligned}
$$

This proves that $\xi_{s}=R_{E}^{\nabla}$.

Corollary 2.33. Let $(L, A)$ be a Lie pair, and $E$ an A-module.

(a) A section $s: E \rightarrow \mathscr{J}_{L / A}^{1} E$ of the short exact sequence (6) is a morphism of $A$ modules if and only if the L-connection it induces on $E$ is compatible with the $A$-action on $E$.

(b) The short exact sequence of A-modules (6) splits if and only if the Atiyah class $\alpha_{E}$ vanishes.

Theorem 2.34. Let $(L, A)$ be a Lie pair, and $E$ an A-module. The natural isomorphism

$$
\operatorname{Ext}_{\mathcal{A}}^{1}\left(E, A^{\perp} \otimes E\right) \stackrel{\cong}{\rightarrow} H^{1}\left(A, A^{\perp} \otimes \text { End } E\right)
$$

maps the extension class of the short exact sequence of A-modules (6) to the Atiyah class of $E$.

We refer the reader to [12, Lemma 8.2.4] for a related result regarding the Atiyah class of dDG algebras, which correspond to the matched pair case as pointed out in Remark 2.6.

\section{LEIBNIZ $\infty$ ALGEBRAS}

In this section, we will explore the rich algebraic structures underlying the Atiyah class of a Lie pair. As we will see in the subsequent discussion, the adequate framework is the notion of Leibniz ${ }_{\infty}$ algebras. Loday's Leibniz ${ }_{\infty}$ algebras [34] are a natural generalization of Stasheff's $L_{\infty}$ algebras [29, 28], where the (skew-)symmetry requirement is dropped.

Throughout this section, we implicitly identify objects of $\mathcal{A}$ to complexes in $\mathcal{A}$ concentrated in degree 0 . Moreover, we make frequent use of the shifting functor: the shift $V[k]$ of a graded vector space $V=\bigoplus_{n} V_{n}$ is determined by the rule $(V[k])_{n}=V_{k+n}$.

We defer most proofs to Section 3.5 .

3.1. $L / A[-1]$ as a Lie algebra object. Recall that a Lie algebra object in a monoidal category $\mathcal{C}$ is an object $\Lambda$ of $\mathcal{C}$ together with a morphism $\lambda \in \operatorname{Hom}_{\mathcal{C}}(\Lambda \otimes \Lambda, \Lambda)$ such that

(a) $\lambda \circ \tau=-\lambda$ (skew-symmetry), and

(b) $\lambda \circ(\mathrm{id} \otimes \lambda)=\lambda \circ(\lambda \otimes \mathrm{id})+\lambda \circ(\mathrm{id} \otimes \lambda) \circ(\tau \otimes \mathrm{id}) \quad$ (Jacobi identity $)$, where $\tau: \Lambda \otimes \Lambda \rightarrow \Lambda \otimes \Lambda$ is the braiding isomorphism. 
Let $(L, A)$ be a Lie pair with quotient $B=L / A$. Note that

$$
\operatorname{Hom}_{D^{+}(\mathcal{A})}(B \otimes B, B[1]) \cong \operatorname{Hom}_{D^{+}(\mathcal{A})}(B[-1] \otimes B[-1], B[-1]) .
$$

Being an element of

$$
\begin{array}{r}
\operatorname{Ext}_{\mathcal{A}}^{1}\left(B, B^{*} \otimes B\right) \cong \operatorname{Ext}_{\mathcal{A}}^{1}(B \otimes B, B) \cong \operatorname{Hom}_{D^{+}(\mathcal{A})}(B \otimes B, B[1]) \cong \\
\operatorname{Hom}_{D^{+}(\mathcal{A})}(B[-1] \otimes B[-1], B[-1]),
\end{array}
$$

the Atiyah class $\alpha_{B}$ of the $A$-module $B$ defines a "Lie bracket" on $B[-1]$. If, moreover, $E$ is an $A$-module, its Atiyah class

$$
\alpha_{E} \in \operatorname{Ext}_{\mathcal{A}}^{1}\left(E, B^{*} \otimes E\right) \cong \operatorname{Ext}_{\mathcal{A}}^{1}(B \otimes E, E) \cong \operatorname{Hom}_{D^{+}(\mathcal{A})}(B[-1] \otimes E[-1], E[-1])
$$

defines a "representation" on $E[-1]$ of the "Lie algebra" $B[-1]$.

More precisely, we have the following theorem, whose proof is deferred to Section 3.5 .

Theorem 3.1. Let $(L, A)$ be a Lie pair with quotient $B=L / A$. Then $B[-1]$ is a Lie algebra object in the derived category $D^{+}(\mathcal{A})$. Moreover, if $E$ is an $A$-module, then $E[-1]$ is a module object over the Lie algebra object $B[-1]$ in the derived category $D^{+}(\mathcal{A})$.

Remark 3.2. From the skew-symmetric property of a Lie algebra, it follows that the Atiyah class $\alpha_{B}$ can indeed be considered as an element in $H^{1}\left(A, S^{2} B^{*} \otimes B\right)$, or more precisely, in the image of the map $H^{1}\left(A, S^{2} B^{*} \otimes B\right) \rightarrow H^{1}\left(A, B^{*} \otimes\right.$ End $\left.B\right)$ induced by the $A$-modules morphism $S^{2} B^{*} \otimes B \rightarrow B^{*} \otimes B^{*} \otimes B\left(\cong B^{*} \otimes\right.$ End $\left.B\right)$.

Remark 3.3. It is implicitly stated in [23] (see also [45, 43]) that, if $X$ is a complex manifold, then $T_{X}[-1]$ is a Lie algebra object in the bounded below derived category $D^{+}(X)$ of coherent sheaves on $X$. This is simply Theorem 3.1 in the special case when $L=T_{X} \otimes \mathbb{C}$ and $A=T_{X}^{0,1}$.

3.2. Jacobi identity up to homotopy. Let $(L, A)$ be a Lie pair and $E$ an $A$-module. The quotient $B=L / A$ is naturally an $A$-module (see Proposition 1.4).

Consider the graded vector spaces

$$
V=\bigoplus_{n=0}^{\infty} \Gamma\left(\wedge^{n} A^{*} \otimes B\right)
$$

and

$$
W=\bigoplus_{n=0}^{\infty} \Gamma\left(\wedge^{n} A^{*} \otimes E\right),
$$

and the covariant differentials

$$
\begin{aligned}
& \partial^{A}: \Gamma\left(\wedge^{\bullet} A^{*} \otimes B\right) \rightarrow \Gamma\left(\wedge^{\bullet+1} A^{*} \otimes B\right) \\
& \partial^{A}: \Gamma\left(\wedge^{\bullet} A^{*} \otimes E\right) \rightarrow \Gamma\left(\wedge^{\bullet+1} A^{*} \otimes E\right)
\end{aligned}
$$

associated to the $A$-actions on $B$ and $E$, respectively. Choosing an $L$-connection $\nabla$ on $L / A$ extending the $A$-action, we obtain the bundle maps $R_{2}: B \otimes B \rightarrow \operatorname{Hom}(A, B)$ and $S_{2}: B \otimes E \rightarrow \operatorname{Hom}(A, E)$ given by

$$
\begin{gathered}
A \ni a \stackrel{R_{2}\left(b_{1}, b_{2}\right)}{\longmapsto} R_{B}^{\nabla}\left(a ; b_{1}\right) b_{2} \in B, \\
A \ni a \stackrel{S_{2}(b, e)}{\longmapsto} R_{E}^{\nabla}(a ; b) e \in E,
\end{gathered}
$$

where $R_{B}^{\nabla}: A \otimes B \rightarrow$ End $B$ and $R_{E}^{\nabla}: A \otimes B \rightarrow$ End $E$ denote the Atiyah cocycles of $B$ and $E$, respectively. 
Theorem 3.4. Up to homotopies, the complex $\left(V[-1], \partial^{A}\right)$ is a differential graded Lie algebra and the complex $\left(W[-1], \partial^{A}\right)$ is a differential graded module over it. The Lie algebra bracket

$$
V[-1] \otimes V[-1] \stackrel{\lambda}{\rightarrow} V[-1]
$$

and the representation

$$
V[-1] \otimes W[-1] \stackrel{\mu}{\rightarrow} W[-1]
$$

are given by

$$
\lambda\left(\left(\xi_{1} \otimes b_{1}\right),\left(\xi_{2} \otimes b_{2}\right)\right)=(-1)^{k_{2}} \xi_{1} \wedge \xi_{2} \wedge R_{2}\left(b_{1}, b_{2}\right)
$$

and

$$
\mu\left(\left(\xi_{1} \otimes b\right),\left(\xi_{2} \otimes e\right)\right)=(-1)^{k_{2}} \xi_{1} \wedge \xi_{2} \wedge S_{2}(b, e),
$$

where $\xi_{1} \in \Gamma\left(\wedge^{k_{1}} A^{*}\right), \xi_{2} \in \Gamma\left(\wedge^{k_{2}} A^{*}\right), b_{1}, b_{2}, b \in \Gamma(B)$, and $e \in \Gamma(E)$.

Consequently, the cohomology $\bigoplus_{i \geq 1} H^{i-1}(A, E)=H^{\bullet}\left(W[-1], \partial^{A}\right)$ is a module over the (graded) Lie algebra $\bigoplus_{i \geq 1} H^{i-1}(A, B)=H^{\bullet}\left(V[-1], \partial^{A}\right)$.

In Section 3.4, we will describe a result which keeps track of higher homotopies.

3.3. Leibniz L $_{\infty}[1]$ algebras. Recall that a graded Leibniz algebra is a $\mathbb{Z}$-graded vector space $V=\bigoplus_{k \in \mathbb{Z}} V_{k}$ equipped with a bilinear bracket $V \otimes V \stackrel{[-,-]}{\longrightarrow} V$ of degree 0 satisfying the graded Leibniz rule

$$
[x,[y, z]]=[[x, y], z]+(-1)^{|x||y|}[y,[x, z]],
$$

for all homogeneous elements $x, y, z \in V$.

If, moreover, $V$ is endowed with a differential $\delta$ of degree 1 satisfying

$$
\delta[x, y]=[\delta x, y]+(-1)^{|x|+1}[x, \delta y]
$$

for all homogeneous elements $x, y \in V$, then we say that $(V,[-,-], \delta)$ is a differential graded Leibniz algebra.

Definition 3.5. A Leibniz $\infty_{\infty}[1]$ algebra is a $\mathbb{Z}$-graded vector space $V=\bigoplus_{n \in \mathbb{Z}} V_{n}$ endowed with a sequence $\left(\lambda_{k}\right)_{k=1}^{\infty}$ of multilinear maps $\lambda_{k}: \otimes^{k} V \rightarrow V$ of degree 1 satisfying the identity

$$
\begin{aligned}
\sum_{1 \leqslant j \leqslant k \leqslant n} & \sum_{\sigma \in \mathfrak{S}_{k-j}^{j-1}} \epsilon\left(\sigma ; v_{1}, \cdots, v_{k-1}\right)(-1)^{\left|v_{\sigma(1)}\right|+\left|v_{\sigma(2)}\right|+\cdots+\left|v_{\sigma(k-j)}\right|} \\
& \lambda_{n-j+1}\left(v_{\sigma(1)}, \cdots, v_{\sigma(k-j)}, \lambda_{j}\left(v_{\sigma(k+1-j)}, \cdots, v_{\sigma(k-1)}, v_{k}\right), v_{k+1}, \cdots, v_{n}\right)=0
\end{aligned}
$$

for each $n \in \mathbb{N}$ and for any homogeneous vectors $v_{1}, v_{2}, \ldots, v_{n} \in V$. Here $\mathfrak{S}_{k-j}^{j-1}$ denotes the set of $(k-j, j-1)$-shuffles ${ }^{1}$, and $\epsilon\left(\sigma ; v_{1}, \cdots, v_{k-1}\right)$ denotes the Koszul sign ${ }^{2}$ of the permutation $\sigma$ of the (homogeneous) vectors $v_{1}, v_{2}, \ldots, v_{k-1}$.

Remark 3.6. If all $\lambda_{k}$ are zero except for $\lambda_{1},\left(V, \lambda_{1}\right)$ is simply a cochain complex. If $\lambda_{k}=0(k \geq 3)$, then $(V[-1],[-,-], d)$ is a graded differential Leibniz algebra, where $[x, y]=(-1)^{|x|} \lambda_{2}(x, y)$, and $d=\lambda_{1}$.

1. A $(k-j, j-1)$-shuffle is a permutation $\sigma$ of the set $\{1,2, \cdots, k-1\}$ such that $\sigma(1) \leqslant \sigma(2) \leqslant \cdots \leqslant$ $\sigma(k-j)$ and $\sigma(k-j+1) \leqslant \sigma(k-j+2) \leqslant \cdots \leqslant \sigma(k-1)$.

2. The Koszul sign of a permutation $\sigma$ of the (homogeneous) vectors $v_{1}, v_{2}, \ldots, v_{n}$ is determined by the relation $v_{\sigma(1)} \odot v_{\sigma(2)} \odot \cdots \odot v_{\sigma(n)}=\epsilon\left(\sigma ; v_{1}, \cdots, v_{n}\right) \cdot v_{1} \odot v_{2} \odot \cdots \odot v_{n}$. 
Remark 3.7. A graded vector space $V$ is a Leibniz $\infty_{\infty}[1]$ algebra if and only if the shifted graded vector space $V[-1]$ is a Leibniz $\infty$ algebra in the sense of Loday [1, 49]. Working with Leibniz $_{\infty}$ [1] algebras rather than Leibniz ${ }_{\infty}$ algebras is convenient, as all maps in the sequence $\left(\lambda_{k}\right)_{k=1}^{n}$ have the same degree in this setting.

Definition 3.8. A module over a Leibniz $\infty_{\infty}[1]$ algebra $V$ is a $\mathbb{Z}$-graded vector space $W=$ $\bigoplus_{n \in \mathbb{Z}} W_{n}$ together with a sequence $\left(\mu_{k}\right)_{k=1}^{\infty}$ of multilinear maps

$$
\mu_{k}:\left(\otimes^{k-1} V\right) \otimes W \rightarrow W
$$

of degree 1 satisfying the identity:

$$
\begin{aligned}
& \sum_{1 \leqslant j \leqslant k \leqslant n-1} \sum_{\sigma \in \mathfrak{S}_{k-j}^{j-1}} \epsilon\left(\sigma ; v_{1}, \cdots, v_{k-1}\right)(-1)^{\left|v_{\sigma(1)}\right|+\left|v_{\sigma(2)}\right|+\cdots+\left|v_{\sigma(k-j)}\right|} \\
& \mu_{n-j+1}\left(v_{\sigma(1)}, \cdots, v_{\sigma(k-j)}, \lambda_{j}\left(v_{\sigma(k+1-j)}, \cdots, v_{\sigma(k-1)}, v_{k}\right), v_{k+1}, \cdots, v_{n-1}, w\right) \\
&+\sum_{1 \leqslant j \leqslant n} \sum_{\sigma \in \mathfrak{S}_{n-j}^{j-1}} \epsilon\left(\sigma ; v_{1}, \cdots, v_{n-1}\right)(-1)^{\left|v_{\sigma(1)}\right|+\left|v_{\sigma(2)}\right|+\cdots+\left|v_{\sigma(n-j)}\right|} \\
& \mu_{n-j+1}\left(v_{\sigma(1)}, \cdots, v_{\sigma(n-j)}, \mu_{j}\left(v_{\sigma(n+1-j)}, \cdots, v_{\sigma(n-1)}, w\right)\right)=0,
\end{aligned}
$$

for each $n \in \mathbb{N}$ and any homogeneous vectors $v_{1}, v_{2}, \ldots, v_{n-1} \in V$ and $w \in W$.

Remark 3.9. A graded vector space $W$ is a module over a Leibniz $\infty_{\infty}[1]$ algebra $V$ if and only if $V \oplus W$ is a Leibniz $\infty_{\infty}[1]$ algebra such that $V$ is a Leibniz $\infty_{\infty}$ [1] subalgebra [27].

The proof of the next proposition is a direct verification, which we omit.

Proposition 3.10. If $\left(V,\left(\lambda_{k}\right)_{k=1}^{\infty}\right)$ is a Leibniz ${ }_{\infty}[1]$ algebra, then $\left(V, \lambda_{1}\right)$ is a cochain complex and its cohomology $H^{\bullet}(V)[-1]$ is a graded Leibniz algebra with bracket $H\left(\lambda_{2}\right)$, the image of $\lambda_{2}$ (seen as a chain map) under the cohomology functor. Moreover, if $\left(W,\left(\mu_{k}\right)_{k=1}^{\infty}\right)$ is a module over $\left(V,\left(\lambda_{k}\right)_{k=1}^{\infty}\right)$, then $\left(W, \mu_{1}\right)$ is a cochain complex and $H\left(\mu_{2}\right)$ is a representation of $H^{\bullet}(V)[-1]$ on the cohomology $H^{\bullet}(W)[-1]$ of $\left(W, \mu_{1}\right)$.

3.4. Main theorem. Unless we state otherwise, we assume throughout this section that $(L, A)$ is a Lie pair and $E$ is an $A$-module. The quotient $B=L / A$ is naturally an $A$-module (see Proposition 1.4). We use the symbol $\partial^{A}$ to denote the covariant differential

$$
\partial^{A}: \Gamma\left(\wedge^{\bullet} A^{*} \otimes\left(\otimes^{\star} B^{*}\right) \otimes E\right) \rightarrow \Gamma\left(\wedge^{\bullet+1} A^{*} \otimes\left(\otimes^{\star} B^{*}\right) \otimes E\right)
$$

associated to the $A$-action on $\left(\otimes^{\star} B^{*}\right) \otimes E$. In particular, for any bundle map $\mu:\left(\wedge^{k} A\right) \otimes$ $\left(\otimes^{l} B\right) \rightarrow B$, we have

$$
\begin{aligned}
\left(\partial^{A} \mu\right)\left(a_{0} \wedge \cdots \wedge a_{k} ; b_{1} \otimes \cdots \otimes b_{l}\right)= & \\
\sum_{i=0}^{k}(-1)^{i}\left\{\nabla_{a_{i}}\left(\mu\left(a_{\vec{i}} ; b_{1} \otimes \cdots \otimes b_{l}\right)\right)-\mu\left(a_{\vec{i}} ; \nabla_{a_{i}}\left(b_{1} \otimes \cdots \otimes b_{l}\right)\right)\right\} & \quad+\sum_{i<j}(-1)^{i+j} \mu\left(\left[a_{i}, a_{j}\right] \wedge a_{\widehat{i, j}} ; b_{1} \otimes \cdots \otimes b_{l}\right),
\end{aligned}
$$

where $a_{\hat{i}}$ stands for $a_{0} \wedge \cdots \wedge \widehat{a_{i}} \wedge \cdots \wedge a_{k}$ and $a_{\widehat{i, j}}$ for $a_{0} \wedge \cdots \wedge \widehat{a_{i}} \wedge \cdots \wedge \widehat{a_{j}} \wedge \cdots \wedge a_{k}$, and $\nabla_{a_{i}}\left(b_{1} \otimes \cdots \otimes b_{l}\right)$ for $\sum_{j=1}^{l} b_{1} \otimes \cdots \otimes \nabla_{a_{i}} b_{j} \otimes \cdots \otimes b_{l}$. 
3.4.1. The operator $\partial^{\nabla}$. Now choose an extension of the $A$-action on $E$ to an $L$-connection $\nabla$ on $E$, an extension of the $A$-action on $B$ to an $L$-connection $\nabla$ on $B$, and a splitting of the short exact sequence of vector bundles

$$
0 \longrightarrow A \stackrel{i}{\longrightarrow} L \stackrel{q}{\longrightarrow} B \longrightarrow 0,
$$

i.e. a pair of maps $j: B \rightarrow L$ and $p: L \rightarrow A$ such that $q \circ j=\operatorname{id}_{B}, p \circ i=\operatorname{id}_{A}$ and $i \circ p+j \circ q=\operatorname{id}_{L}$ :

$$
0 \rightleftarrows A \underset{p}{\stackrel{i}{\rightleftarrows}} L \stackrel{q}{\rightleftarrows} B \underset{j}{\rightleftarrows} B
$$

This splitting determines a map

$$
\Gamma(B) \times \Gamma(A) \rightarrow \Gamma(A):(b, a) \mapsto p[j(b), i(a)],
$$

which we will denote by $\Delta$ since it satisfies the relations

$$
\Delta_{f b} a=f \Delta_{b} a \quad \text { and } \quad \Delta_{b}(f a)=\left\langle\rho^{*} d f, j(b)\right\rangle a+f \Delta_{b} a,
$$

for all $f \in C^{\infty}(M), b \in \Gamma(B)$, and $a \in \Gamma(A)$. In some sense, $B$ "acts" on $A$.

Identifying sections of $\wedge^{\bullet} A^{*} \otimes\left(\otimes^{\star} B^{*}\right) \otimes E$ with bundle maps $\wedge^{\bullet} A \otimes\left(\otimes^{\star} B\right) \rightarrow E$, we define a differential operator

$$
\partial^{\nabla}: \Gamma\left(\wedge^{k} A^{*} \otimes\left(\otimes^{l} B^{*}\right) \otimes E\right) \rightarrow \Gamma\left(\wedge^{k} A^{*} \otimes\left(\otimes^{l+1} B^{*}\right) \otimes E\right)
$$

by

$$
(-1)^{k} \iota_{b_{0}}\left(\partial^{\nabla} \omega\right)=\nabla_{j\left(b_{0}\right)} \omega
$$

or, more precisely,

$$
\begin{aligned}
& (-1)^{k}\left(\partial^{\nabla} \omega\right)\left(a_{1}, \cdots, a_{k} ; b_{0}, \cdots, b_{l}\right)=\nabla_{j\left(b_{0}\right)}\left(\omega\left(a_{1}, \cdots, a_{k} ; b_{1}, \cdots, b_{l}\right)\right) \\
& -\omega\left(\Delta_{b_{0}} a_{1}, \cdots, a_{k} ; b_{1}, \cdots, b_{l}\right)-\cdots-\omega\left(a_{1}, \cdots, \Delta_{b_{0}} a_{k} ; b_{1}, \cdots, b_{l}\right) \\
& \quad-\omega\left(a_{1}, \cdots, a_{k} ; \nabla_{j\left(b_{0}\right)} b_{1}, \cdots, b_{l}\right)-\cdots-\omega\left(a_{1}, \cdots, a_{k} ; b_{1}, \cdots, \nabla_{j\left(b_{0}\right)} b_{l}\right),
\end{aligned}
$$

where $a_{1}, \cdots, a_{k} \in \Gamma(A), b_{0}, \cdots, b_{l} \in \Gamma(B)$, and $\omega: \wedge^{k} A \otimes(\otimes l B) \rightarrow E$.

Note that $\partial^{\nabla}$ depends on the choice of the $L$-connections extending the $A$-actions and the splitting $j: B \rightarrow L$ of the short exact sequence $(23)$, while $\partial^{A}$ does not.

The chosen splitting of (23) does also determine three vector bundle maps

$$
\alpha: \wedge^{2} B \rightarrow A, \quad \beta: \wedge^{2} B \rightarrow B, \quad \text { and } \quad \Omega: \wedge^{2} B \rightarrow \text { End } B
$$

given by

$$
\begin{gathered}
\alpha\left(b_{1}, b_{2}\right)=p\left[j\left(b_{1}\right), j\left(b_{2}\right)\right] \\
\beta\left(b_{1}, b_{2}\right)=\nabla_{j\left(b_{1}\right)} b_{2}-\nabla_{j\left(b_{2}\right)} b_{1}-q\left[j\left(b_{1}\right), j\left(b_{2}\right)\right]
\end{gathered}
$$

and

$$
\Omega\left(b_{1}, b_{2}\right)=\nabla_{j\left(b_{1}\right)} \nabla_{j\left(b_{2}\right)}-\nabla_{j\left(b_{2}\right)} \nabla_{j\left(b_{1}\right)}-\nabla_{\left[j\left(b_{1}\right), j\left(b_{2}\right)\right]} .
$$

Proposition 3.11. For any $a \in \Gamma(A)$ and $b_{1}, b_{2} \in \Gamma(B)$, we have

$$
R_{B}^{\nabla}\left(a ; b_{1}\right) b_{2}-R_{B}^{\nabla}\left(a ; b_{2}\right) b_{1}=\left(\nabla_{a} \beta\right)\left(b_{1}, b_{2}\right)
$$

or, equivalently,

$$
R_{2}\left(b_{1}, b_{2}\right)-R_{2}\left(b_{2}, b_{1}\right)=\left(\partial^{A} \beta\right)\left(b_{1}, b_{2}\right) .
$$


Proof. For convenience, we set $\widetilde{b}=j(b), \forall b \in \Gamma(B)$. Hence $[a, \widetilde{b}]=-\Delta_{b} a+\widetilde{\nabla_{a} b}$ and

$$
\left.\left[\widetilde{b_{1}}, \widetilde{b_{2}}\right]=\alpha\left(b_{1}, b_{2}\right)+\widetilde{\nabla_{\widetilde{b_{1}}} b_{2}}-\widetilde{\nabla_{\widetilde{b_{2}}} b_{1}}-\widetilde{\beta\left(b_{1}, b_{2}\right.}\right) .
$$

A straightforward computation yields the equality

$$
\begin{aligned}
q\left(\left[\left[a, \widetilde{b_{1}}\right], \widetilde{b_{2}}\right]\right. & \left.+\left[\left[\widetilde{b_{1}}, \widetilde{b_{2}}\right], a\right]+\left[\left[\widetilde{b_{2}}, a\right], \widetilde{b_{1}}\right]\right) \\
& =R_{B}^{\nabla}\left(a ; b_{2}\right) b_{1}-R_{B}^{\nabla}\left(a ; b_{1}\right) b_{2}+\nabla_{a}\left(\beta\left(b_{1}, b_{2}\right)\right)-\beta\left(\nabla_{a} b_{1}, b_{2}\right)-\beta\left(b_{1}, \nabla_{a} b_{2}\right) .
\end{aligned}
$$

The result follows from the Jacobi identity of the Lie algebroid $L$.

Note that, since $R_{B}^{\nabla}$ is (by its very definition) independent of the choice of the splitting, Proposition 3.11 asserts that, unlike $\beta, \partial^{A} \beta$ does not depend on the choice of splitting.

3.4.2. The maps $R_{n}$. Recall the bundle map $R_{2}: B \otimes B \rightarrow \operatorname{Hom}(A, B)$ associated to the Atiyah cocycle of $B$ given by 20 . Since $B$ is an $A$-module, we can substitute $B$ for $E$ in Equation (24) and define a sequence of bundle maps

$$
R_{n}: \otimes^{n} B \rightarrow \operatorname{Hom}(A, B)
$$

inductively by the relation

$$
R_{n+1}=\partial^{\nabla} R_{n}, \quad \text { for } n \geq 2 .
$$

Hence, we have

$$
R_{n+1}\left(b_{0} \otimes b_{1} \otimes \cdots \otimes b_{n}\right)=R_{n}\left(\nabla_{j\left(b_{0}\right)}\left(b_{1} \otimes \cdots \otimes b_{n}\right)\right)-\nabla_{j\left(b_{0}\right)}\left(R_{n}\left(b_{1} \otimes \cdots \otimes b_{n}\right)\right) .
$$

Example 3.12. Let $L=A \bowtie B$ be a matched pair of Lie algebras. Any bilinear map $\gamma: B \otimes B \rightarrow B$ determines an $L$-connection $\nabla$ on $B$ extending its $A$-module structure (and conversely): $\nabla_{b_{1}} b_{2}=\gamma\left(b_{1}, b_{2}\right)$. Taking $\gamma=0$, the Atiyah cocycle reads

$$
R_{B}^{\nabla}\left(a ; b_{1}\right) b_{2}=\nabla_{\Delta_{b_{1}} a} b_{2} \text {. }
$$

Hence

$$
R_{n}\left(b_{1}, b_{2}, b_{3}, \cdots, b_{n}\right)=\nabla_{\Delta_{b_{n-1}} \Delta_{b_{n-2}} \cdots \Delta_{b_{1}}(-)} b_{n}
$$

3.4.3. Leibniz $z_{\infty}[1]$ algebra (and modules) arising from a Lie pair. Consider the sequence of $k$-ary operations $\lambda_{k}: \otimes^{k} V \rightarrow V(k \in \mathbb{N})$ on the graded vector space

$$
V=\bigoplus_{n=0}^{\infty} \Gamma\left(\wedge^{n} A^{*} \otimes B\right)
$$

defined by $\lambda_{1}=\partial^{A}$ and, for $k \geq 2$,

$$
\lambda_{k}\left(\xi_{1} \otimes b_{1}, \cdots, \xi_{k} \otimes b_{k}\right)=(-1)^{\left|\xi_{1}\right|+\cdots+\left|\xi_{k}\right|} \xi_{1} \wedge \cdots \wedge \xi_{k} \wedge R_{k}\left(b_{1}, \cdots, b_{k}\right),
$$

where $b_{1}, \ldots, b_{k} \in \Gamma(B)$ and $\xi_{1}, \ldots, \xi_{k}$ are homogeneous elements in $\Gamma\left(\wedge^{\bullet} A^{*}\right)$.

We are now ready to state the main result of the paper.

Theorem 3.13. When endowed with the sequence of multibrackets $\left(\lambda_{k}\right)_{k \in \mathbb{N}}$ defined above, the graded vector space $V=\bigoplus_{n=0}^{\infty} \Gamma\left(\wedge^{n} A^{*} \otimes B\right)$ becomes a Leibniz $\infty_{\infty}[1]$ algebra.

Similarly, we can introduce the bundle map $S_{2}: B \otimes E \rightarrow \operatorname{Hom}(A, E)$ given by

$$
A \ni a \stackrel{S_{2}(b ; e)}{\longmapsto} R_{E}^{\nabla}(a ; b) \cdot e \in E,
$$

where $R_{E}^{\nabla}: A \otimes B \rightarrow$ End $E$ denotes the Atiyah cocycle of the $A$-module $E$, and then define a sequence of bundle maps

$$
S_{n}:\left(\otimes^{n-1} B\right) \otimes E \rightarrow \operatorname{Hom}(A, E)
$$


inductively by the relation

$$
S_{n+1}=\partial^{\nabla} S_{n}, \quad \text { for } n \geq 2 .
$$

Consider the graded vector space $W=\bigoplus_{n=0}^{\infty} \Gamma\left(\wedge^{n} A^{*} \otimes E\right)$ and the sequence of $k$-ary brackets $\mu_{k}:\left(\otimes^{k-1} V\right) \otimes W \rightarrow W(k \in \mathbb{N})$ defined by $\mu_{1}=\partial^{A}$ and, for $k \geq 2$,

$$
\mu_{k}\left(\xi_{1} \otimes b_{1}, \cdots, \xi_{k-1} \otimes b_{k-1} ; \xi_{k} \otimes e\right)=(-1)^{\left|\xi_{1}\right|+\cdots+\left|\xi_{k}\right|} \xi_{1} \wedge \cdots \wedge \xi_{k} \wedge S_{k}\left(b_{1}, \cdots, b_{k-1} ; e\right),
$$

where $b_{1}, \ldots, b_{k-1} \in \Gamma(B), e \in \Gamma(E)$, and $\xi_{1}, \ldots, \xi_{k}$ are homogeneous elements of $\Gamma\left(\wedge^{\bullet} A^{*}\right)$.

Theorem 3.14. When endowed with the sequence of multibrackets $\left(\mu_{k}\right)_{k \in \mathbb{N}}$ defined above, the graded vector space $W=\bigoplus_{n=0}^{\infty} \Gamma\left(\wedge^{n} A^{*} \otimes E\right)$ becomes a Leibniz ${ }_{\infty}[1]$ module over the Leibniz $_{\infty}[1]$ algebra $\left(V,\left(\lambda_{k}\right)_{k \in \mathbb{N}}\right)$.

Example 3.15. A Lie bialgebra $\left(\mathfrak{g}, \mathfrak{g}^{*}\right)$ is a matched pair of Lie algebras. Therefore, it induces two Lie pairs: $\left(\mathfrak{g} \bowtie \mathfrak{g}^{*}, \mathfrak{g}\right)$ and $\left(\mathfrak{g} \bowtie \mathfrak{g}^{*}, \mathfrak{g}^{*}\right)$. It follows from Example 3.12 and Theorem 3.13 that both $\bigoplus_{n \geq 0} \wedge^{n} \mathfrak{g}^{*} \otimes \mathfrak{g}^{*}$ and $\bigoplus_{n \geq 0} \wedge^{n} \mathfrak{g} \otimes \mathfrak{g}$ are Leibniz ${ }_{\infty}$ [1] algebras.

Let $A$ be a Lie algebroid over a manifold $M$. By an $A$-algebra, we mean a bundle (of finite or infinite rank) of associative algebras $\mathcal{C}$ over $M$, which is an $A$-module, and on which $\Gamma(A)$ acts by derivations. For a commutative $A$-algebra $\mathcal{C}$, the sequence of maps $\left(\lambda_{k}\right)_{k \in \mathbb{N}}$ extends, in a natural way, to the graded vector space $\bigoplus_{n=0}^{\infty} \Gamma\left(\wedge^{n} A^{*} \otimes B \otimes \mathcal{C}\right)$. Similarly, the sequence of maps $\left(\mu_{k}\right)_{k \in \mathbb{N}}$ extends to the graded space $\bigoplus_{n=0}^{\infty} \Gamma\left(\wedge^{n} A^{*} \otimes E \otimes \mathcal{C}\right)$.

Theorem 3.16. Let $(L, A)$ be a Lie pair with quotient $B$, and let $\mathcal{C}$ be a commutative $A$-algebra. When endowed with the sequence of multibrackets $\left(\lambda_{k}\right)_{k \in \mathbb{N}}$, the graded vector space $\Gamma\left(\wedge^{\bullet} A^{*} \otimes B \otimes \mathcal{C}\right)$ becomes a Leibniz $z_{\infty}[1]$ algebra. Moreover, if $E$ is an A-module, the graded vector space $\Gamma\left(\wedge^{\bullet} A^{*} \otimes E \otimes \mathcal{C}\right)[-1]$ becomes a Leibniz $z_{\infty}[1]$ module over the Leibniz $z_{\infty}[1]$ algebra $\Gamma\left(\wedge^{\bullet} A^{*} \otimes B \otimes \mathcal{C}\right)[-1]$.

As an immediate consequence, we have the following

Corollary 3.17. Under the same hypothesis as in Theorem 3.16, $\bigoplus_{i \geq 1} H^{i-1}(A, B \otimes \mathcal{C})$ is a graded Lie algebra, and $\bigoplus_{i \geq 1} H^{i-1}(A, E \otimes \mathcal{C})$ a module over it.

Example 3.18. Let $\mathfrak{g}$ be a Lie subalgebra of a Lie algebra $\mathfrak{d}$ as in Example2.10. Assume that $\mathcal{C}$ is a commutative $\mathfrak{g}$-algebra.

Every linear map $\boldsymbol{L}: \mathfrak{d} \rightarrow \operatorname{End}(\mathfrak{d} / \mathfrak{g})$ that extends the $\mathfrak{g}$-module structure $\mathfrak{g} \rightarrow \operatorname{End}(\mathfrak{d} / \mathfrak{g})$ induces a 2-ary bracket on $\wedge^{\bullet-1} \mathfrak{g}^{*} \otimes \mathfrak{d} / \mathfrak{g} \otimes \mathcal{C}$ :

$$
\left[\xi_{1} \otimes b_{1} \otimes c_{1}, \xi_{2} \otimes b_{2} \otimes c_{2}\right]=(-1)^{\left|\xi_{2}\right|} \xi_{1} \wedge \xi_{2} \otimes\left(\partial^{\mathfrak{g}} \boldsymbol{L}\right)\left(-; b_{1}\right) \cdot b_{2} \otimes c_{1} c_{2},
$$

which in turn induces a (graded) Lie algebra bracket on the Chevalley-Eilenberg cohomology $\bigoplus H^{\bullet-1}(\mathfrak{g}, \mathfrak{d} / \mathfrak{g} \otimes \mathcal{C})$. Here $\xi_{i} \otimes b_{i} \otimes c_{i}(i=1,2)$ are cocycles with $\xi_{1}, \xi_{2} \in \wedge^{\bullet} \mathfrak{g}^{*}$, $b_{1}, b_{2} \in \mathfrak{d} / \mathfrak{g}$ and $c_{1}, c_{2} \in \mathcal{C}$.

Moreover, if $E$ is a $\mathfrak{g}$-module, every linear map $M: \mathfrak{d} \rightarrow$ End $E$ that extends the $\mathfrak{g}$-module structure $\mathfrak{g} \rightarrow$ End $E$ gives rise to a bilinear map

$$
\left(\xi_{1} \otimes b \otimes c_{1}\right) \triangleright\left(\xi_{2} \otimes e \otimes c_{2}\right)=(-1)^{\left|\xi_{2}\right|} \xi_{1} \wedge \xi_{2} \otimes\left(\partial^{\mathfrak{g}} \boldsymbol{M}\right)(-; b) \cdot e \otimes c_{1} c_{2},
$$

which induces a representation on $\bigoplus H^{\bullet-1}(\mathfrak{g}, E \otimes \mathcal{C})$ of the graded Lie algebra $\bigoplus H^{\bullet-1}(\mathfrak{g}, \mathfrak{d} / \mathfrak{g} \otimes$ $\mathcal{C})$. Here $\xi_{1} \otimes b \otimes c_{1}$ and $\xi_{2} \otimes e \otimes c_{2}$ are cocycles with $\xi_{1}, \xi_{2} \in \wedge^{\bullet} \mathfrak{g}^{*}, b \in \mathfrak{d} / \mathfrak{g}, e \in E$ and $c_{1}, c_{2} \in \mathcal{C}$.

Take a complement $\mathfrak{h}$ of $\mathfrak{g}$ in $\mathfrak{d}$ so that we can write $\mathfrak{d}=\mathfrak{g} \oplus \mathfrak{h}$. Then $\mathfrak{d} / \mathfrak{g}$ can be identified with $\mathfrak{h}$, on which the $\mathfrak{h}$-action is given by $a \cdot h=\operatorname{pr}_{\mathfrak{h}}[a, h]$. Take $\boldsymbol{L}: \mathfrak{d} \rightarrow$ End $\mathfrak{h}$ to be the trivial extension of the $\mathfrak{g}$-module structure $\mathfrak{g} \rightarrow$ End $\mathfrak{h}$, i.e. set $\left.\boldsymbol{L}\right|_{\mathfrak{h}}=0$. Then the 2-ary bracket in Equation (31) is given by

$$
\left[f \otimes c_{1}, g \otimes c_{2}\right]=[f, g] \otimes c_{1} c_{2},
$$


where $f$ is a linear map from $\wedge^{p} \mathfrak{g}$ to $\mathfrak{h}, g$ is a linear map from $\wedge^{q} \mathfrak{g}$ to $\mathfrak{h}, c_{1}, c_{2} \in \mathcal{C}$, and $[f, g]$ is a linear map from $\wedge^{p+q+1} \mathfrak{g}$ to $\mathfrak{h}$ given by

$$
\begin{aligned}
{[f, g]\left(a_{0}, a_{1}, \cdots, a_{p+q}\right)=} & \\
& -\sum_{\sigma \in \mathfrak{S}_{1, p, q}} \operatorname{sgn}(\sigma) \operatorname{pr}_{\mathfrak{h}}\left[\operatorname{pr}_{\mathfrak{g}}\left[a_{\sigma(0)}, f\left(a_{\sigma(1)}, \cdots, a_{\sigma(p)}\right)\right], g\left(a_{\sigma(p+1)}, \cdots, a_{\sigma(p+q)}\right)\right] .
\end{aligned}
$$

Here $\mathfrak{S}_{1, p, q}$ is the set of all permutations $\sigma$ of $\{0,1, \cdots, p+q\}$ satisfying $\sigma(1)<\cdots<\sigma(p)$ and $\sigma(p+1)<\cdots<\sigma(p+q)$.

Remark 3.19. It is natural to ask how the Leibniz ${ }_{\infty}[1]$ algebra structure obtained in Theorem 3.13 and the Leibniz ${ }_{\infty}$ [1] module structure in Theorem 3.14 depend on the choice of connections and the splitting data. This question will be investigated somewhere else.

\subsection{4. $L_{\infty}$ rather than Leibniz $z_{\infty}$.}

Theorem 3.20. Let $(A, B)$ be a matched pair of Lie algebroids with direct sum $L=A \bowtie B$. Assume there exists a flat torsion free $B$-connection on $B$. Then the maps $R_{n}$ defined as in Equation (29) are totally symmetric, the multibrackets $\lambda_{k}: \otimes^{k} V \rightarrow V(k \in \mathbb{N})$ defined as in Equation (30) on the graded vector space $V=\bigoplus_{n=0}^{\infty} \Gamma\left(\wedge^{n} A^{*} \otimes B\right)$ are graded symmetric, and $V[-1]$ is actually an $L_{\infty}$ algebra.

The following example is due to Camille Laurent-Gengoux.

Example 3.21. The general Lie algebra $\mathfrak{g l}_{n}(\mathbb{C})$ decomposes as the direct sum of the unitary Lie algebra $\mathfrak{u}_{n}$ and the Lie algebra $\mathfrak{t}_{n}$ of upper triangular matrices with real diagonal coefficients. Both $\mathfrak{u}_{n}$ and $\mathfrak{t}_{n}$ are isotropic with respect to the natural nondegenerate adinvariant inner product

$$
X \otimes Y \mapsto \operatorname{im}(\operatorname{tr}(X Y))
$$

on $\mathfrak{g l}_{n}(\mathbb{C})$. Hence $\left(\mathfrak{u}_{n}, \mathfrak{t}_{n}\right)$ is a matched pair of Lie algebras as well as a Lie bialgebra. Matrix multiplication being associative, setting $\nabla_{X} Y=X Y$ for any $X, Y \in \mathfrak{t}_{n}$ defines a flat torsion free $\mathfrak{t}_{n}$-connection on $\mathfrak{t}_{n}$. It follows from Theorem 3.20 that $\Gamma\left(\wedge \mathfrak{u}_{n}^{*} \otimes \mathfrak{t}_{n}\right)[-1] \cong$ $\Gamma\left(\wedge \mathfrak{t}_{n} \otimes \mathfrak{t}_{n}\right)[-1]$ is an $L_{\infty}$ algebra.

As an application of Theorem 3.20, consider a Kähler manifold $X$. The complexification $\nabla^{\mathbb{C}}$ of its Levi-Civita connection is a $T_{X} \otimes \mathbb{C}$-connection on $T_{X} \otimes \mathbb{C}$. Set $A=T_{X}^{0,1}$ and $B=T_{X}^{1,0}$. Then $(A, B)$ is a matched pair of Lie algebroids, whose direct sum $A \bowtie B$ is isomorphic, as a Lie algebroid, to $T_{X} \otimes \mathbb{C}$. It is easy to see that $\nabla^{\mathbb{C}}$ induces a flat torsion free $B$-connection on $B$. In this context, the tensors $R_{n} \in \Omega^{0,1}\left(\operatorname{Hom}\left(\otimes^{n} T_{X}^{1,0}, T_{X}^{1,0}\right)\right)$ are the curvature $R_{2} \in \Omega^{1,1}\left(\right.$ End $\left.T_{X}^{1,0}\right) \cong \Omega^{0,2}\left(\operatorname{Hom}\left(T_{X}^{1,0} \otimes T_{X}^{1,0}, T_{X}^{1,0}\right)\right)$ and its higher covariant derivatives: $R_{i+1}=\partial^{\nabla} R_{i}$. Applying Theorem 3.20, we recover a result of Kapranov [23]:

Corollary 3.22 (Kapranov). The shifted Dolbeault complex $\Omega^{0, \bullet-1}\left(T_{X}^{1,0}\right)$ of a Kähler manifold $X$ is an $L_{\infty}$ algebra. The $n$-th multibracket

$$
\lambda_{n}: \Omega^{0, j_{1}}\left(T_{X}^{1,0}\right) \otimes \cdots \otimes \Omega^{0, j_{n}}\left(T_{X}^{1,0}\right) \rightarrow \Omega^{0, j_{1}+\cdots+j_{n}+1}\left(T_{X}^{1,0}\right)
$$

is the composition of the wedge product

$$
\Omega^{0, j_{1}}\left(T_{X}^{1,0}\right) \otimes \cdots \otimes \Omega^{0, j_{n}}\left(T_{X}^{1,0}\right) \rightarrow \Omega^{0, j_{1}+\cdots+j_{n}}\left(\otimes^{n} T_{X}^{1,0}\right)
$$

with the map

$$
\Omega^{0, j_{1}+\cdots+j_{n}}\left(\otimes^{n} T_{X}^{1,0}\right) \rightarrow \Omega^{0, j_{1}+\cdots+j_{n}+1}\left(T_{X}^{1,0}\right)
$$

associated to $R_{n} \in \Omega^{0,1}\left(\operatorname{Hom}\left(\otimes^{n} T_{X}^{1,0}, T_{X}^{1,0}\right)\right)$ in the obvious way.

3.5. Proofs. This part is devoted to the proofs of the theorems claimed earlier in this section. For convenience, we set $\widetilde{b}=j(b), \forall b \in \Gamma(B)$. 
3.5.1. Atiyah class as a Lie bracket. Below we follow the notations as introduced in Section 3.4.1.

Lemma 3.23. For any $a_{1}, a_{2} \in \Gamma(A)$ and $b \in \Gamma(B)$, we have

$$
\left[\Delta_{b} a_{1}, a_{2}\right]+\left[a_{1}, \Delta_{b} a_{2}\right]-\Delta_{b}\left[a_{1}, a_{2}\right]=\Delta_{\nabla_{a_{1}} b} a_{2}-\Delta_{\nabla_{a_{2} b} b} a_{1} .
$$

Proof. We have

$$
\begin{aligned}
& \left.p\left(\left[\tilde{b}, a_{1}\right], a_{2}\right]+\left[\left[a_{1}, a_{2}\right], \widetilde{b}\right]+\left[\left[a_{2}, \widetilde{b}\right], a_{1}\right]\right) \\
= & \left.p\left[p\left[\widetilde{b}, a_{1}\right]+q \widetilde{[b}, a_{1}\right], a_{2}\right]-p\left[\widetilde{b},\left[a_{1}, a_{2}\right]\right]+p\left[p\left[a_{2}, \widetilde{b}\right]+q\left[a_{2}, \widetilde{b}\right], a_{1}\right] \\
= & {\left.\left[p\left[\widetilde{b}, a_{1}\right], a_{2}\right]-p\left[\widetilde{q\left[a_{1}, \widetilde{b}\right.}\right], a_{2}\right]-p\left[\widetilde{b},\left[a_{1}, a_{2}\right]\right]+\left[a_{1}, p\left[\widetilde{b}, a_{2}\right]\right]+p\left[q\left[a_{2}, \widetilde{b}\right], a_{1}\right] } \\
= & {\left[\Delta_{b} a_{1}, a_{2}\right]-\Delta_{\nabla_{a_{1}} b} a_{2}-\Delta_{b}\left[a_{1}, a_{2}\right]+\left[a_{1}, \Delta_{b} a_{2}\right]+\Delta_{\nabla_{a_{2}} b} a_{1} . }
\end{aligned}
$$

The result follows from the Jacobi identity of the Lie algebroid $L$.

Note that a bundle map $\omega:\left(\wedge^{k} A\right) \otimes\left(\otimes^{l} B\right) \rightarrow E$ determines a bundle map

$$
\overleftarrow{\omega}: \wedge^{k} A \rightarrow\left(\otimes B^{*}\right) \otimes E
$$

and vice versa.

Lemma 3.24. For any bundle map $\omega:\left(\wedge^{k} A\right) \otimes\left(\otimes^{l} B\right) \rightarrow E$, any $a_{0}, \ldots, a_{k} \in \Gamma(A)$ and any $b_{0}, \ldots, b_{l} \in \Gamma(B)$, we have

$$
\begin{aligned}
& (-1)^{k}\left(\partial^{A} \partial^{\nabla} \omega+\partial^{\nabla} \partial^{A} \omega\right)\left(a_{0}, \cdots, a_{k} ; b_{0}, \ldots, b_{l}\right) \\
& \quad=\sum_{i=0}^{k}(-1)^{i}\left\langle\nabla_{a_{i}} \nabla_{\widetilde{b_{0}}}\left(\overleftarrow{\omega}\left(\widehat{a_{i}}\right)\right)-\nabla_{\widetilde{b_{0}}} \nabla_{a_{i}}\left(\overleftarrow{\omega}\left(\widehat{a_{i}}\right)\right)-\nabla_{\left[a_{i}, \widetilde{b_{0}}\right]}\left(\overleftarrow{\omega}\left(\widehat{a_{i}}\right)\right) \mid b_{1} \otimes \cdots \otimes b_{l}\right\rangle \\
& \quad=\sum_{i=0}^{k}(-1)^{i}\left\{R_{E}^{\nabla}\left(a_{i} ; b_{0}\right) \cdot \omega\left(\widehat{a_{i}} ; \widehat{b_{0}}\right)-\sum_{j=1}^{l} \omega\left(\widehat{a_{i}} ; b_{1}, \cdots, R_{B}^{\nabla}\left(a_{i} ; b_{0}\right) \cdot b_{j}, \cdots, b_{l}\right)\right\}
\end{aligned}
$$

where $\widehat{a_{i}}$ stands for $a_{0} \wedge \cdots \wedge a_{i-1} \wedge a_{i+1} \wedge \cdots \wedge a_{k}$ and $\widehat{b_{0}}$ for $b_{1} \otimes \cdots \otimes b_{l}$.

Sketch of proof. The first equality follows from a cumbersome computation at the last step of which use is made of Lemma 3.23. The second equality is immediate.

Given $\mu \in \Gamma\left(\left(\wedge^{k_{1}} A^{*}\right) \otimes\left(\otimes^{l_{1}} B^{*}\right) \otimes B\right), \nu \in \Gamma\left(\left(\wedge^{k_{2}} A^{*}\right) \otimes\left(\otimes^{l_{2}} B^{*}\right) \otimes B\right)$, and arbitrary sections $b_{1}, \ldots, b_{l_{1}}, b_{1}^{\prime}, \ldots, b_{l_{2}}^{\prime}$ of $B$, let

$$
\left\lceil\mu\left(b_{1}, \cdots, b_{i-1}, \nu\left(b_{1}^{\prime}, \cdots, b_{l_{2}}^{\prime}\right), b_{i+1}, \cdots, b_{l_{1}}\right)\right\rfloor \in \Gamma\left(\wedge^{k_{1}+k_{2}} A^{*} \otimes B\right),
$$

be defined, as a bundle map $\wedge^{k_{1}+k_{2}} A \rightarrow B$, sending $a_{1} \wedge \cdots \wedge a_{k_{1}+k_{2}}$ to

$$
\begin{aligned}
\sum_{\sigma \in \mathfrak{S}_{k_{1}}^{k_{2}}} \operatorname{sgn}(\sigma) \mu\left(a_{\sigma(1)}, \cdots, a_{\sigma\left(k_{1}\right)} ; b_{1}, \cdots, b_{i-1},\right. & \\
& \left.\nu\left(a_{\sigma\left(k_{1}+1\right)}, \cdots, a_{\sigma\left(k_{1}+k_{2}\right)} ; b_{1}^{\prime}, \cdots, b_{l_{2}}^{\prime}\right), b_{i+1}, \cdots, b_{l_{1}}\right) .
\end{aligned}
$$

In particular, if $\mu=\alpha_{1} \otimes \beta_{1} \otimes u$ and $\nu=\alpha_{2} \otimes \beta_{2} \otimes v$ with $\alpha_{1} \in \Gamma\left(\wedge^{k_{1}} A^{*}\right), \alpha_{2} \in \Gamma\left(\wedge^{k_{2}} A^{*}\right)$, $\beta_{1} \in \Gamma\left(\otimes^{l_{1}} B^{*}\right), \beta_{2} \in \Gamma\left(\otimes^{l_{2}} B^{*}\right)$, and $u, v \in \Gamma(B)$, then

$$
\begin{aligned}
\left\lceil\mu \left( b_{1}, \cdots, b_{i-1}, \nu\left(b_{1}^{\prime}, \cdots,\right.\right.\right. & \left.\left.\left.b_{l_{2}}^{\prime}\right), b_{i+1}, \cdots, b_{l_{1}}\right)\right\rfloor= \\
& \beta_{1}\left(b_{1}, \cdots, b_{i-1}, v, b_{i+1}, \cdots, b_{l_{1}}\right) \beta_{2}\left(b_{1}^{\prime}, \cdots, b_{l_{2}}^{\prime}\right) \cdot\left(\alpha_{1} \wedge \alpha_{2}\right) \otimes u .
\end{aligned}
$$


Corollary 3.25. For any $n \geq 2$ and $b_{0}, \ldots, b_{n} \in \Gamma(B)$, we have

$$
\begin{aligned}
-\left(\left(\partial^{A} \partial^{\nabla}+\partial^{\nabla} \partial^{A}\right) R_{n}\right)\left(b_{0}, \cdots, b_{n}\right) & =\left\lceil R_{2}\left(b_{0}, R_{n}\left(b_{1}, \cdots, b_{n}\right)\right)\right\rfloor \\
& +\sum_{j=1}^{n}\left\lceil R_{n}\left(b_{1}, \cdots, b_{j-1}, R_{2}\left(b_{0}, b_{j}\right), b_{j+1}, \cdots, b_{n}\right)\right\rfloor .
\end{aligned}
$$

Proof. Apply Lemma 3.24 to $\omega=R_{n}$.

Corollary 3.26. For any $b_{0}, b_{1}, b_{2} \in \Gamma(B)$, we have

$$
\begin{aligned}
-\left(\partial^{A} R_{3}\right)\left(b_{0}, b_{1}, b_{2}\right)=\left\lceil R_{2}\left(b_{0}, R_{2}\left(b_{1}, b_{2}\right)\right)\right\rfloor+\left\lceil R_{2}\left(R_{2}\left(b_{0}, b_{1}\right), b_{2}\right)\right\rfloor \\
+\left\lceil R_{2}\left(b_{1}, R_{2}\left(b_{0}, b_{2}\right)\right)\right\rfloor .
\end{aligned}
$$

Proof. Since $\partial^{A} R_{2}=0$ and $\partial^{\nabla} R_{2}=R_{3}$, taking $n=2$ in Corollary 3.25 yields the result.

Sketch of proof of Theorem 3.1. The interchange isomorphism $\tau: B[-1] \otimes B[-1] \rightarrow B[-1] \otimes$ $B[-1]$ is the image in $D^{+}(\mathcal{A})$ of the chain map $\tau: B[-1] \otimes B[-1] \rightarrow B[-1] \otimes B[-1]$ given by $\tau\left(b_{1} \otimes b_{2}\right)=-b_{2} \otimes b_{1}, \forall b_{1}, b_{2} \in B$ - the negative sign is due to $B[-1]$ being a complex concentrated in degree 1 (see Equation (15)). Recall that $R_{2}$ is a cocycle (w.r.t. $\partial^{A}$ ). Its cohomology class $\alpha_{B}$, the Atiyah class of $B$, can be seen as an element of $\operatorname{Hom}_{D^{+}(\mathcal{A})}(B[-1] \otimes B[-1], B[-1])$. Proposition 3.11 implies the equality $\alpha_{B} \circ \tau=-\alpha_{B}$ in $\operatorname{Hom}_{D^{+}(\mathcal{A})}(B[-1] \otimes B[-1], B[-1])$. Corollary 3.26 implies that the Jacobi identity $\alpha_{B} \circ\left(\mathrm{id} \otimes \alpha_{B}\right)=\alpha_{B} \circ\left(\alpha_{B} \otimes \mathrm{id}\right)+\alpha_{B} \circ\left(\mathrm{id} \otimes \alpha_{B}\right) \circ(\tau \otimes \mathrm{id})$ holds in $D^{+}(\mathcal{A})$. Indeed, each of the terms in the right hand side of Equation (33) can be interpreted as a Yoneda product, a composition of morphisms in the derived category.

3.5.2. Jacobi identity up to homotopy. Consider the cochain complex $\left(V[-1], \partial^{A}\right)$, where the graded vector space $V=\bigoplus_{k=0}^{\infty} V_{k}$ is given by $V_{k}=\Gamma\left(\wedge^{k} A^{*} \otimes B\right)$ so that, if $\xi \in \Gamma\left(\wedge^{k} A^{*}\right)$ and $b \in \Gamma(B)$, then $\xi \otimes b \in(V[-1])_{k+1}$.

Lemma 3.27. The graded linear map $\lambda: V[-1] \otimes V[-1] \rightarrow V[-1]$ given by

$$
\lambda\left(v_{1} \otimes v_{2}\right)=(-1)^{k_{2}} \xi_{1} \wedge \xi_{2} \wedge R_{2}\left(b_{1}, b_{2}\right)
$$

for any $v_{1}=\xi_{1} \otimes b_{1} \in(V[-1])_{k_{1}+1}$ and $v_{2}=\xi_{2} \otimes b_{2} \in(V[-1])_{k_{2}+1}$ is a chain map.

Proof. A straightforward computation yields

$$
\left(\partial^{A} \circ \lambda-\lambda \circ \partial^{A}\right)\left(v_{1} \otimes v_{2}\right)=(-1)^{k_{1}} \xi_{1} \wedge \xi_{2} \wedge\left(\partial^{A} R_{2}\right)\left(b_{1}, b_{2}\right) .
$$

The result follows from $\partial^{A} R_{2}=0$ (see Theorem 2.5 and the definition 20 of $R_{2}$ ).

Now, consider the interchange isomorphism $\tau: V[-1] \otimes V[-1] \rightarrow V[-1] \otimes V[-1]$ given by $\tau\left(v_{1} \otimes v_{2}\right)=(-1)^{\left|v_{1}\right|\left|v_{2}\right|} v_{2} \otimes v_{1}$.

Lemma 3.28. The chain map $\lambda$ is skew-symmetric up to homotopy:

$$
\lambda+\lambda \circ \tau=\partial^{A} \circ \Theta+\Theta \circ \partial^{A},
$$

where the graded map $\Theta: V[-1] \otimes V[-1] \rightarrow V[-2]$ is given by

$$
\Theta\left(v_{1} \otimes v_{2}\right)=(-1)^{k_{1}} \xi_{1} \wedge \xi_{2} \otimes \beta\left(b_{1}, b_{2}\right)
$$

for any $v_{1}=\xi_{1} \otimes b_{1} \in(V[-1])_{k_{1}+1}$ and $v_{2}=\xi_{2} \otimes b_{2} \in(V[-1])_{k_{2}+1}$. 
Proof. Straightforward computations yield

$$
(\lambda+\lambda \circ \tau)\left(v_{1} \otimes v_{2}\right)=(-1)^{k_{2}} \xi_{1} \wedge \xi_{2} \wedge\left(R_{2}\left(b_{1}, b_{2}\right)-R_{2}\left(b_{2}, b_{1}\right)\right)
$$

and

$$
\left(\partial^{A} \circ \Theta+\Theta \circ \partial^{A}\right)\left(v_{1} \otimes v_{2}\right)=(-1)^{k_{2}} \xi_{1} \wedge \xi_{2} \wedge\left(\left(\partial^{A} \beta\right)\left(b_{1}, b_{2}\right)\right)
$$

The result follows from Proposition 3.11 .

Lemma 3.29. The chain map $\lambda$ satisfies the Jacobi identity up to a homotopy:

$$
-\lambda \circ(\mathrm{id} \otimes \lambda)+\lambda \circ(\lambda \otimes \mathrm{id})+\lambda \circ(\mathrm{id} \otimes \lambda) \circ(\tau \otimes \mathrm{id})=\partial^{A} \circ \Xi+\Xi \circ \partial^{A},
$$

where the graded map $\Xi: V[-1] \otimes V[-1] \otimes V[-1] \rightarrow V[-2]$ is given by

$$
\Xi\left(v_{0} \otimes v_{1} \otimes v_{2}\right)=(-1)^{k_{0}+k_{2}} \xi_{0} \wedge \xi_{1} \wedge \xi_{2} \wedge R_{3}\left(b_{0}, b_{1}, b_{2}\right)
$$

for any $v_{i}=\xi_{i} \otimes b_{i} \in(V[-1])_{k_{i}+1}$ with $i \in\{0,1,2\}$.

Proof. Straightforward computations yield

$$
\begin{gathered}
(\lambda \circ(\mathrm{id} \otimes \lambda))\left(v_{0} \otimes v_{1} \otimes v_{2}\right)=(-1)^{k_{1}} \xi_{0} \wedge \xi_{1} \wedge \xi_{2} \wedge\left\lceil R_{2}\left(b_{0}, R_{2}\left(b_{1}, b_{2}\right)\right)\right\rfloor, \\
(\lambda \circ(\lambda \otimes \mathrm{id}))\left(v_{0} \otimes v_{1} \otimes v_{2}\right)=-(-1)^{k_{1}} \xi_{0} \wedge \xi_{1} \wedge \xi_{2} \wedge\left\lceil R_{2}\left(R_{2}\left(b_{0}, b_{1}\right), b_{2}\right)\right\rfloor, \\
(\lambda \circ(\mathrm{id} \otimes \lambda) \circ(\tau \otimes \mathrm{id}))\left(v_{0} \otimes v_{1} \otimes v_{2}\right)=-(-1)^{k_{1}} \xi_{0} \wedge \xi_{1} \wedge \xi_{2} \wedge\left\lceil R_{2}\left(b_{1}, R_{2}\left(b_{0}, b_{2}\right)\right)\right\rfloor,
\end{gathered}
$$

and

$$
\left(\partial^{A} \circ \Theta+\Theta \circ \partial^{A}\right)\left(v_{0} \otimes v_{1} \otimes v_{2}\right)=(-1)^{k_{1}} \xi_{0} \wedge \xi_{1} \wedge \xi_{2} \wedge\left(\left(\partial^{A} R_{3}\right)\left(b_{0}, b_{1}, b_{2}\right)\right) .
$$

The result follows from Corollary 3.26 .

Theorem 3.4 immediately follows from Lemmas 3.27, 3.28, and 3.29,

Note that Theorem 3.4 could also be seen as a corollary of Theorems 3.13 and 3.14 .

3.5.3. Leibniz ${ }_{\infty}[1]$ algebra (and modules) arising from a Lie pair.

Lemma 3.30. For any $n \geq 3$ and any arbitrary sections $b_{1}, \ldots, b_{n}$ of $B$, we have

$$
\begin{gathered}
-\left(\partial^{A} R_{n}\right)\left(b_{1}, \ldots, b_{n}\right)=\sum_{\substack{i+j=n+1 \\
i \geq 2 \\
j \geq 2}} \sum_{k=j}^{n} \sum_{\sigma \in \mathfrak{S}_{k-j}^{j-1}} \\
\left\lceil R_{i}\left(b_{\sigma(1)}, \cdots, b_{\sigma(k-j)}, R_{j}\left(b_{\sigma(k+1-j)}, \cdots, b_{\sigma(k-1)}, b_{k}\right), b_{k+1}, \cdots, b_{n}\right)\right\rfloor .
\end{gathered}
$$

Proof. We reason by induction. The formula holds for $n=3$ by Corollary 3.26 . Assuming the formula holds for $n=N$, we get

$$
\begin{aligned}
& \left(\partial^{\nabla} \partial^{A} R_{N}\right)\left(b_{0}, \ldots, b_{N}\right)=\left(\nabla_{b_{0}}\left(\partial^{A} R_{N}\right)\right)\left(b_{1}, \ldots, b_{N}\right)=\sum_{\substack{i+j=N \\
i \geq 2 \\
j \geq 2}} \sum_{k=j}^{N} \sum_{\substack{j \in \mathfrak{S}_{k-j}^{j-1} \\
k-1}} \\
& \left\{\left\lceil R_{i+1}\left(b_{0}, b_{\sigma(1)}, \cdots, b_{\sigma(k-j)}, R_{j}\left(b_{\sigma(k+1-j)}, \cdots, b_{\sigma(k-1)}, b_{k}\right), b_{k+1}, \cdots, b_{n}\right)\right\rfloor\right. \\
& \left.+\left\lceil R_{i}\left(b_{\sigma(1)}, \cdots, b_{\sigma(k-j)}, R_{j+1}\left(b_{0}, b_{\sigma(k+1-j)}, \cdots, b_{\sigma(k-1)}, b_{k}\right), b_{k+1}, \cdots, b_{n}\right)\right\rfloor\right\} .
\end{aligned}
$$

Observing that

$$
\partial^{A} R_{N+1}=\left(\partial^{A} \partial^{\nabla}+\partial^{\nabla} \partial^{A}\right) R_{N}-\partial^{\nabla} \partial^{A} R_{N}
$$

and recalling Corollary 3.25 , it is easy to check that the desired formula holds for $n=N+1$ as well. 
Lemma 3.31. For any bundle map $\omega:\left(\wedge^{k} A\right) \otimes\left(\otimes^{l} B\right) \rightarrow B$ and any $b_{1}, \ldots, b_{l} \in \Gamma(B)$, we have

$$
\partial^{A}\left(\overleftarrow{\omega}\left(b_{1}, \ldots, b_{l}\right)\right)-\left(\overleftarrow{\partial^{A} \omega}\right)\left(b_{1}, \ldots, b_{l}\right)=(-1)^{k} \sum_{j=0}^{l}\left[\omega\left(b_{1}, \cdots, b_{j-1}, \partial^{A} b_{j}, b_{j+1}, \cdots, b_{l}\right)\right\rfloor,
$$

where $\overleftarrow{\omega}$ is defined by Equation 32

Proof. For any $a_{0}, \ldots, a_{k} \in \Gamma(A)$, we have

$$
\begin{aligned}
& \left\langle\partial^{A}\left(\overleftarrow{\omega}\left(b_{1}, \ldots, b_{l}\right)\right)-\left(\overleftarrow{\partial^{A} \omega}\right)\left(b_{1}, \ldots, b_{l}\right) \mid a_{0} \wedge \cdots \wedge a_{k}\right\rangle \\
= & \sum_{j=0}^{l} \sum_{i=0}^{k}(-1)^{i} \omega\left(a_{0}, \cdots, a_{i-1}, a_{i+1}, \cdots, a_{k} ; b_{1}, \cdots, \nabla_{a_{i}} b_{j}, \cdots, b_{l}\right) \\
= & (-1)^{k} \sum_{j=0}^{l} \sum_{\sigma \in \mathfrak{S}_{k}^{1}} \operatorname{sgn}(\sigma) \omega\left(a_{\sigma(0)}, \cdots, a_{\sigma(k-1)} ; b_{1}, \cdots, \nabla_{a_{\sigma(k)}} b_{j}, \cdots, b_{l}\right) \\
= & (-1)^{k} \sum_{j=0}^{l}\left\langle\left\lceil\omega\left(b_{1}, \cdots, b_{j-1}, \partial^{A} b_{j}, b_{j+1}, \cdots, b_{l}\right)\right\rfloor \mid a_{0} \wedge \cdots \wedge a_{k}\right\rangle .
\end{aligned}
$$

Proof of Theorem 3.13. We only need to check that the generalized Leibniz identity (22) holds. Since $\lambda_{1}=\partial^{A}$ and $\left(\partial^{A}\right)^{2}=0$, Equation $(22)$ is obviously true for $n=1$. Let $n \geq 2$ and $v_{i}=\xi_{i} \otimes b_{i} \in \Gamma\left(\wedge^{p_{i}} A^{*} \otimes B\right)$ for all $i \in\{1, \ldots, n\}$. The l.h.s. of $(22)$ is

$$
\begin{aligned}
\sum_{1 \leqslant j \leqslant k \leqslant n} \sum_{\sigma \in \mathfrak{S}_{k-j}^{j-1}} \epsilon\left(\sigma ; v_{1}, \cdots, v_{k-1}\right)(-1)^{\left|v_{\sigma(1)}\right|+\left|v_{\sigma(2)}\right|+\cdots+\left|v_{\sigma(k-j)}\right|} \\
\lambda_{n-j+1}\left(v_{\sigma(1)}, \cdots, v_{\sigma(k-j)}, \lambda_{j}\left(v_{\sigma(k+1-j)}, \cdots, v_{\sigma(k-1)}, v_{k}\right), v_{k+1}, \cdots, v_{n}\right) .
\end{aligned}
$$

Separating the terms involving $\lambda_{1}$ (aka $\partial^{A}$ ) from the others, it can be rewritten as

$$
\begin{gathered}
\partial^{A}\left(\lambda_{n}\left(v_{1}, \cdots, v_{n}\right)\right)+\sum_{\substack{i+j=n+1 \\
i \geq 2 \\
j \geq 2}} \sum_{k=j}^{n} \sum_{\sigma \in \mathfrak{S}_{k-j}^{j-1}} \epsilon\left(\sigma ; \xi_{1}, \cdots, \xi_{k-1}\right)(-1)^{p_{\sigma(1)}+\cdots+p_{\sigma(k-j)}} \\
\lambda_{i}\left(v_{\sigma(1)}, \cdots, v_{\sigma(k-j)}, \lambda_{j}\left(v_{\sigma(k+1-j)}, \cdots, v_{\sigma(k-1)}, v_{k}\right), v_{k+1}, \cdots, v_{n}\right) \\
+\sum_{k=1}^{n}(-1)^{p_{1}+\cdots+p_{k-1}} \lambda_{n}\left(v_{1}, \cdots, v_{k-1},\left(\partial^{A} \xi_{k}\right) \otimes b_{k}+(-1)^{p_{k}} \xi_{k} \otimes\left(\partial^{A} b_{k}\right), v_{k+1}, \cdots, v_{n}\right) .
\end{gathered}
$$

Since each $\lambda_{k}$ is given by Equation 30 in terms of the corresponding $R_{k}$, it in turn becomes

$$
\begin{aligned}
& \partial^{A}\left((-1)^{p_{1}+\cdots+p_{n}} \xi_{1} \wedge \cdots \wedge \xi_{n} \wedge R_{n}\left(b_{1}, \cdots, b_{n}\right)\right) \\
& +\sum_{\substack{i+j=n+1 \\
i \geq 2 \\
j \geq 2}} \sum_{k=j}^{n} \sum_{\sigma \in \mathfrak{S}_{k-j}^{j-1}} \epsilon\left(\sigma ; \xi_{1}, \cdots, \xi_{k-1}\right) \xi_{\sigma(1)} \wedge \cdots \wedge \xi_{\sigma(k-1)} \wedge \xi_{k} \wedge \cdots \wedge \xi_{n} \wedge \\
& \left\lceil R_{i}\left(b_{\sigma(1)}, \cdots, b_{\sigma(k-j)}, R_{j}\left(b_{\sigma(k+1-j)}, \cdots, b_{\sigma(k-1)}, b_{k}\right), b_{k+1}, \cdots, b_{n}\right)\right\rfloor \\
& +\sum_{k=1}^{n}(-1)^{1+p_{k}+p_{k+1}+\cdots+p_{n}} \xi_{1} \wedge \cdots \wedge \xi_{k-1} \wedge \partial^{A} \xi_{k} \wedge \xi_{k+1} \wedge \cdots \wedge \xi_{n} \wedge R_{n}\left(b_{1}, \cdots, b_{n}\right)
\end{aligned}
$$




$$
+\sum_{k=1}^{n} \xi_{1} \wedge \cdots \wedge \xi_{n} \wedge\left\lceil R_{n}\left(b_{1}, \cdots, b_{k-1}, \partial^{A} b_{k}, b_{k+1}, \cdots, b_{n}\right)\right\rfloor
$$

which simplifies to

$$
\begin{aligned}
\xi_{1} \wedge \cdots \wedge & \xi_{n} \wedge\left\{\partial^{A}\left(R_{n}\left(b_{1}, \cdots, b_{n}\right)\right)+\sum_{\substack{i+j=n+1 \\
i \geq 2 \\
j \geq 2}} \sum_{k=j}^{n} \sum_{\sigma \in \mathfrak{S}_{k-j}^{j-1}}\right. \\
\left\lceil R_{i}\left(b_{\sigma(1)}, \cdots, b_{\sigma(k-j)}, R_{j}\left(b_{\sigma(k+1-j)}, \cdots, b_{\sigma(k-1)}, b_{k}\right), b_{k+1}, \cdots, b_{n}\right)\right\rfloor & \\
& \left.+\sum_{k=1}^{n}\left\lceil R_{n}\left(b_{1}, \cdots, b_{k-1}, \partial^{A} b_{k}, b_{k+1}, \cdots, b_{n}\right)\right\rfloor\right\} .
\end{aligned}
$$

The result now follows from Lemmas 3.30 and 3.31 .

The proofs of Theorem 3.14, Theorem 3.16 and Corollary 3.17 go along the same line mutatis mutandis.

3.5.4. $L_{\infty}$ rather than Leibniz $z_{\infty}$.

Lemma 3.32. For any $b_{0}, b_{1}, b_{2} \in \Gamma(B)$, we have

$$
R_{3}\left(b_{0}, b_{1}, b_{2}\right)-R_{3}\left(b_{1}, b_{0}, b_{2}\right)=R_{2}\left(\beta\left(b_{0}, b_{1}\right), b_{2}\right)-\left(\partial^{A} \Omega\right)\left(b_{0}, b_{1}\right) \cdot b_{2},
$$

where $\beta: \wedge^{2} B \rightarrow B$ and $\Omega: \wedge^{2} B \rightarrow$ End $B$ are bundle maps as introduced in Equations (26) -27 .

Proof. The second Bianchi identity $d^{\nabla} R^{\nabla}=0$ holds for the curvature $R^{\nabla}: \wedge^{2} L \rightarrow$ End $B$ of the $L$-connection $\nabla$ on $B$ extending the $A$-action. Hence, for any $a \in \Gamma(A)$ and $b_{0}, b_{1}, b_{2} \in \Gamma(B)$, we have

$$
\begin{aligned}
0= & \left(d^{\nabla} R^{\nabla}\right)\left(a, \widetilde{b}_{0}, \widetilde{b}_{1}\right) \\
= & \nabla_{a}\left(R^{\nabla}\left(\widetilde{b}_{0}, \widetilde{b}_{1}\right)\right)-\nabla_{\widetilde{b}_{0}}\left(R^{\nabla}\left(a, \widetilde{b}_{1}\right)\right)+\nabla_{\widetilde{b}_{1}}\left(R^{\nabla}\left(a, \widetilde{b}_{0}\right)\right) \\
& -R^{\nabla}\left(\left[a, \widetilde{b}_{0}\right], \widetilde{b}_{1}\right)+R^{\nabla}\left(\left[a, \widetilde{b}_{1}\right], \widetilde{b}_{0}\right)-R^{\nabla}\left(\left[\widetilde{b}_{0}, \widetilde{b}_{1}\right], a\right) \\
= & \nabla_{a}\left(R^{\nabla}\left(\widetilde{b}_{0}, \widetilde{b}_{1}\right)\right)-\nabla_{\widetilde{b}_{0}}\left(R^{\nabla}\left(a, \widetilde{b}_{1}\right)\right)+\nabla_{\widetilde{b}_{1}}\left(R^{\nabla}\left(a, \widetilde{b}_{0}\right)\right) \\
& -R^{\nabla}\left(\widetilde{\nabla_{a} b_{0}}, \widetilde{b}_{1}\right)+R^{\nabla}\left(\Delta_{b_{0}} a, \widetilde{b}_{1}\right)+R^{\nabla}\left(\widetilde{\nabla_{a} b_{1}}, \widetilde{b}_{0}\right)-R^{\nabla}\left(\Delta_{b_{1}} a, \widetilde{b}_{0}\right) \\
& \left.-R^{\nabla}\left(\alpha\left(b_{0}, b_{1}\right), a\right)-R^{\nabla}\left(\widetilde{\nabla_{\widetilde{b}_{0}} b_{1}}, a\right)+R^{\nabla}\left(\widetilde{\nabla_{\widetilde{b}_{1}} b_{0}}, a\right)+R^{\nabla}\left(\widetilde{\beta\left(b_{0}, b_{1}\right.}\right), a\right)
\end{aligned}
$$

and thus

$$
\begin{aligned}
0= & \left(d^{\nabla} R^{\nabla}\right)\left(a, \widetilde{b}_{0}, \widetilde{b}_{1}\right) \cdot b_{2} \\
= & \left(\partial^{\nabla} R_{B}^{\nabla}\right)\left(a ; b_{0}, b_{1}\right) \cdot b_{2}-\left(\partial^{\nabla} R_{B}^{\nabla}\right)\left(a ; b_{1}, b_{0}\right) \cdot b_{2}-R_{B}^{\nabla}\left(a, \beta\left(b_{0}, b_{1}\right)\right) \cdot b_{2} \\
& +\nabla_{a}\left(R^{\nabla}\left(\widetilde{b}_{0}, \widetilde{b}_{1}\right)\right) \cdot b_{2}-R^{\nabla}\left(\widetilde{\nabla_{a} b_{0}}, \widetilde{b}_{1}\right) \cdot b_{2}-R^{\nabla}\left(\widetilde{b}_{0}, \widetilde{\nabla_{a} b_{1}}\right) \cdot b_{2}
\end{aligned}
$$

or, equivalently,

$$
0=R_{3}\left(b_{0}, b_{1}, b_{2}\right)-R_{3}\left(b_{1}, b_{0}, b_{2}\right)-R_{2}\left(\beta\left(b_{0}, b_{1}\right), b_{2}\right)+\left(\partial^{A} \Omega\right)\left(b_{0}, b_{1}\right) \cdot b_{2} .
$$

Lemma 3.33. For any $a \in \Gamma(A)$ and $b_{0}, b_{1} \in \Gamma(B)$, we have

$$
\left[\alpha\left(b_{0}, b_{1}\right), a\right]+\alpha\left(\nabla_{a} b_{0}, b_{1}\right)+\alpha\left(b_{0}, \nabla_{a} b_{1}\right)=\Delta_{b_{0}} \Delta_{b_{1}} a-\Delta_{b_{1}} \Delta_{b_{0}} a-\Delta_{q\left[\widetilde{b}_{0}, \widetilde{b}_{1}\right]} a .
$$


Proof. We have

$$
\begin{aligned}
& p\left(\left[\widetilde{b}_{1},\left[\widetilde{b}_{0}, a\right]\right]+\left[\widetilde{b}_{0},\left[a, \widetilde{b}_{1}\right]\right]+\left[a,\left[\widetilde{b}_{1}, \widetilde{b}_{0}\right]\right]\right) \\
= & p\left[\widetilde{b}_{1}, p\left[\widetilde{b}_{0}, a\right]\right]+p\left[\widetilde{b}_{0}, p\left[a, \widetilde{b_{1}}\right]\right]+p\left[a, p\left[\widetilde{b}_{1}, \widetilde{b}_{0}\right]\right] \\
& +p\left[\widetilde{b_{1}}, q\left[\widetilde{\widetilde{b}_{0}}, a\right]\right]+p\left[\widetilde{b}_{0}, \widetilde{\left.q\left[a, \widetilde{b}_{1}\right]\right]}+p\left[a, q \widetilde{\left.q\left[\widetilde{b}_{1}, \widetilde{b}_{0}\right]\right]}\right.\right. \\
= & \Delta_{b_{1}} \Delta_{b_{0}} a-\Delta_{b_{0}} \Delta_{b_{1}} a+p\left[a, \alpha\left(b_{1}, b_{0}\right)\right]+p\left[\widetilde{\nabla_{a} b_{0}}, \widetilde{b}_{1}\right]+p\left[\widetilde{b}_{0}, \widetilde{\nabla_{a} b_{1}}\right]+p\left[q\left[\widetilde{b}_{0}, \widetilde{b}_{1}\right], a\right] \\
= & \Delta_{b_{1}} \Delta_{b_{0}} a-\Delta_{b_{0}} \Delta_{b_{1}} a+\left[\alpha\left(b_{0}, b_{1}\right), a\right]+\alpha\left(\nabla_{a} b_{0}, b_{1}\right)+\alpha\left(b_{0}, \nabla_{a} b_{1}\right)+\Delta \widetilde{q\left[\widetilde{b}_{0}, \widetilde{b}_{1}\right]} a .
\end{aligned}
$$

The result follows from the Jacobi identity of $L$.

Lemma 3.34. For any $n \geq 3, a \in \Gamma(A)$ and $b_{0}, b_{1}, \ldots, b_{n} \in \Gamma(B)$, we have

$$
\begin{aligned}
& R_{n+1}\left(a ; b_{0}, b_{1}, b_{2}, \cdots, b_{n}\right)-R_{n+1}\left(a ; b_{1}, b_{0}, b_{2}, \cdots, b_{n}\right)= \\
& \Omega\left(b_{0}, b_{1}\right) \cdot R_{n-1}\left(a ; b_{2}, \cdots, b_{n}\right)-\sum_{j=2}^{n} R_{n-1}\left(a ; b_{2}, \cdots, \Omega\left(b_{0}, b_{1}\right) \cdot b_{j}, \cdots, b_{n}\right) \\
& +\nabla_{\alpha\left(b_{0}, b_{1}\right)}\left(R_{n-1}\left(a ; b_{2}, \cdots, b_{n}\right)\right)-\sum_{j=2}^{n} R_{n-1}\left(a ; b_{2}, \cdots, \nabla_{\alpha\left(b_{0}, b_{1}\right)} b_{j}, \cdots, b_{n}\right) \\
& -R_{n-1}\left(\left[\alpha\left(b_{0}, b_{1}\right), a\right]+\alpha\left(\nabla_{a} b_{0}, b_{1}\right)+\alpha\left(b_{0}, \nabla_{a} b_{1}\right) ; b_{2}, \cdots, b_{n}\right) \\
& +R_{n}\left(a ; \beta\left(b_{0}, b_{1}\right), b_{2}, \cdots, b_{n}\right) .
\end{aligned}
$$

Here $R_{k}\left(a ; b_{1}, \cdots, b_{k}\right)$ means $R_{k}\left(b_{1}, \cdots, b_{k}\right)(a)$.

Sketch of proof. Straightforward computation at the last step of which use is made of Lemma 3.33 .

Proposition 3.35. Let $L=A \bowtie B$ be a matched pair of Lie algebroids endowed with a flat torsion free $B$-connection on $B$. (These data determine a splitting of the short exact sequence of vector bundles $0 \rightarrow A \rightarrow L \rightarrow B \rightarrow 0$ and an $L$-connection on $B$ extending the $A$-action such that the three associated bundle maps $\alpha, \beta$, and $\Omega$ are trivial.) Then each $R_{n}: \otimes^{n} B \rightarrow \operatorname{Hom}(A, B)$ is totally symmetric in its $n$ arguments.

Proof. It follows from Proposition 3.11 and Lemma 3.32 that $R_{2}$ and $R_{3}$ are invariant under the permutation of their first two arguments. By Lemma 3.34, the same property holds for all higher $R_{n}$. Moreover, it is easy to see that, if $R_{n}$ is symmetric in its $n$ arguments, then $R_{n+1}$ is symmetric in its last $n$ arguments since $R_{n+1}=\partial^{\nabla} R_{n}$. The result follows by induction.

Theorem 3.20 , which says that $V[-1]$ is an $L_{\infty}$-algebra when the assumptions of Proposition 3.35 are satisfied, is an immediate consequence of Proposition 3.35 and Theorem 3.13 .

\section{REFERENCES}

1. Mourad Ammar and Norbert Poncin, Coalgebraic approach to the Loday infinity category, stem differential for 2n-ary graded and homotopy algebras, Ann. Inst. Fourier (Grenoble) 60 (2010), no. 1, 355-387. MR 2664318 (2011i:16017)

2. Dima Arinkin and Andrei Căldăraru, When is the self-intersection of a subvariety a fibration?, Adv. Math. 231 (2012), no. 2, 815-842. MR 2955193

3. Michael Francis Atiyah, Complex analytic connections in fibre bundles, Trans. Amer. Math. Soc. 85 (1957), 181-207. MR 0086359 (19,172c)

4. Dror Bar-Natan, Stavros Garoufalidis, Lev Rozansky, and Dylan P. Thurston, Wheels, wheeling, and the Kontsevich integral of the unknot, Israel J. Math. 119 (2000), 217-237. MR 1802655 (2002a:57014) 
5. Dror Bar-Natan, Thang T. Q. Le, and Dylan P. Thurston, Two applications of elementary knot theory to Lie algebras and Vassiliev invariants, Geom. Topol. 7 (2003), 1-31 (electronic). MR 1988280 (2004f:57017)

6. Martin Bordemann, (Bi)modules, morphisms, and reduction of star-products: the symplectic case, foliations, and obstructions, Travaux mathématiques. Fasc. XVI, Trav. Math., XVI, Univ. Luxemb., Luxembourg, 2005, pp. 9-40. MR 2223149 (2007f:53122)

7. Atiyah classes and equivariant connections on homogeneous spaces, Travaux mathématiques. Fasc. XX, Trav. Math., XX, Univ. Luxemb., Luxembourg, 2012, pp. 29-82. MR 3014184

8. Raoul Bott, Lectures on characteristic classes and foliations, Lectures on algebraic and differential topology (Second Latin American School in Math., Mexico City, 1971), Springer, Berlin, 1972, Notes by Lawrence Conlon, with two appendices by J. Stasheff, pp. 1-94. Lecture Notes in Math., Vol. 279. MR 0362335 (50 \#14777)

9. Damien Calaque, A PBW theorem for inclusions of (sheaves of) Lie algebroids, Rend. Semin. Mat. Univ. Padova 131 (2014), 23-47. MR 3217749

10. Damien Calaque, Andrei Căldăraru, and Junwu Tu, PBW for an inclusion of Lie algebras, J. Algebra 378 (2013), 64-79. MR 3017014

11. Damien Calaque and Carlo A. Rossi, Lectures on Duflo isomorphisms in Lie algebra and complex geometry, EMS Series of Lectures in Mathematics, European Mathematical Society (EMS), Zürich, 2011. MR 2816610

12. Damien Calaque and Michel Van den Bergh, Hochschild cohomology and Atiyah classes, Adv. Math. 224 (2010), no. 5, 1839-1889. MR 2646112 (2011i:14037)

13. Zhuo Chen, Mathieu Stiénon, and Ping Xu, A Hopf algebra associated with a Lie pair, C. R. Math. Acad. Sci. Paris 352 (2014), no. 11, 929-933. MR 3268765

14. Kevin Costello, A geometric construction of the Witten genus, I, Proceedings of the International Congress of Mathematicians. Volume II, Hindustan Book Agency, New Delhi, 2010, pp. 942-959. MR 2827826

15. __ A geometric construction of the Witten genus, II, arXiv:1112.0816 (2011).

16. Andrei Căldăraru and Simon Willerton, The Mukai pairing. I. A categorical approach, New York J. Math. 16 (2010), 61-98. MR 2657369 (2011g:18012)

17. Vasiliy Dolgushev, Dmitry Tamarkin, and Boris Tsygan, Formality of the homotopy calculus algebra of hochschild (co)chains, arXiv:0807.5117 (2008).

18. _ Formality theorems for Hochschild complexes and their applications, Lett. Math. Phys. 90 (2009), no. 1-3, 103-136. MR 2565036 (2011b:53221)

19. Darij Grinberg, Poincaré-Birkhoff-Witt type results for inclusions of Lie algebras, Master's thesis, Massachusetts Institute of Technology, Boston, MA, 2011.

20. Philip J. Higgins and Kirill. C. H. Mackenzie, Algebraic constructions in the category of Lie algebroids, J. Algebra 129 (1990), no. 1, 194-230. MR 1037400 (92e:58241)

21. Johannes Hübschmann, Poisson cohomology and quantization, J. Reine Angew. Math. 408 (1990), 57-113. MR 1058984 (92e:17027)

22. André Joyal and Ross Street, Braided tensor categories, Adv. Math. 102 (1993), no. 1, 20-78. MR 1250465 (94m:18008)

23. Mikhail Kapranov, Rozansky-Witten invariants via Atiyah classes, Compositio Math. 115 (1999), no. 1, 71-113. MR 1671737 (2000h:57056)

24. Maxim Kontsevich, Operads and motives in deformation quantization, Lett. Math. Phys. 48 (1999), no. 1, 35-72, Moshé Flato (1937-1998). MR 1718044 (2000j:53119)

25. _ Rozansky-Witten invariants via formal geometry, Compositio Math. 115 (1999), no. 1, 115127. MR 1671725 (2000h:57057)

26. _ Deformation quantization of Poisson manifolds, Lett. Math. Phys. 66 (2003), no. 3, $157-216$. MR 2062626 (2005i:53122)

27. Tom Lada, $L_{\infty}$ algebra representations, Appl. Categ. Structures 12 (2004), no. 1, 29-34, Homotopy theory. MR 2057409 (2005f:18013)

28. Tom Lada and Martin Markl, Strongly homotopy Lie algebras, Comm. Algebra 23 (1995), no. 6, 2147-2161. MR 1327129 (96d:16039)

29. Tom Lada and Jim Stasheff, Introduction to SH Lie algebras for physicists, Internat. J. Theoret. Phys. 32 (1993), no. 7, 1087-1103. MR 1235010 (94g:17059) 
30. Camille Laurent-Gengoux, Mathieu Stiénon, and Ping Xu, Holomorphic Poisson manifolds and holomorphic Lie algebroids, Int. Math. Res. Not. IMRN (2008), Art. ID rnn 088, 46. MR 2439547 (2009i:53082)

31. __ Exponential map and $L_{\infty}$ algebra associated to a Lie pair, C. R. Math. Acad. Sci. Paris 350 (2012), no. 17-18, 817-821. MR 2989383

32. _ Poincaré-Birkhoff-Witt isomorphisms and Kapranov dg-manifolds, arXiv:1408.2903 (2014).

33. Camille Laurent-Gengoux and Yannick Voglaire, Invariant connections and PBW theorem for Lie groupoid pairs, arXiv:1507.01051 (2015).

34. Jean-Louis Loday, Une version non commutative des algèbres de Lie: les algèbres de Leibniz, Enseign. Math. (2) 39 (1993), no. 3-4, 269-293. MR 1252069 (95a:19004)

35. Jiang-Hua Lu, Poisson homogeneous spaces and Lie algebroids associated to Poisson actions, Duke Math. J. 86 (1997), no. 2, 261-304. MR 1430434 (98d:58204)

36. Kirill. C. H. Mackenzie and Tahar Mokri, Locally vacant double Lie groupoids and the integration of matched pairs of Lie algebroids, Geom. Dedicata 77 (1999), no. 3, 317-330. MR 1716681 (2000k:58028)

37. Kirill C. H. Mackenzie and Ping Xu, Classical lifting processes and multiplicative vector fields, Quart. J. Math. Oxford Ser. (2) 49 (1998), no. 193, 59-85. MR 1617335 (99m:58210)

38. Nikita Markarian, The Atiyah class, Hochschild cohomology and the Riemann-Roch theorem, J. Lond. Math. Soc. (2) 79 (2009), no. 1, 129-143. MR 2472137 (2010d:14020)

39. Rajan Amit Mehta, Mathieu Stiénon, and Ping Xu, The Atiyah class of a dg-vector bundle, C. R. Math. Acad. Sci. Paris 353 (2015), no. 4, 357-362. MR 3319134

40. Tahar Mokri, Matched pairs of Lie algebroids, Glasgow Math. J. 39 (1997), no. 2, 167-181. MR 1460632 (99a:58165)

41. Pierre Molino, Classe d'Atiyah d'un feuilletage et connexions transverses projetables., C. R. Acad. Sci. Paris Sér. A-B 272 (1971), A779-A781. MR 0281224 (43 \#6943)

42. __ Propriétés cohomologiques et propriétés topologiques des feuilletages à connexion transverse projetable, Topology 12 (1973), 317-325. MR 0353332 (50 \#5816)

43. Ajay C. Ramadoss, The big Chern classes and the Chern character, Internat. J. Math. 19 (2008), no. 6, 699-746. MR 2431634 (2010h:14028)

44. George S. Rinehart, Differential forms on general commutative algebras, Trans. Amer. Math. Soc. 108 (1963), 195-222. MR 0154906 (27 \#4850)

45. Justin Roberts and Simon Willerton, On the Rozansky-Witten weight systems, Algebr. Geom. Topol. 10 (2010), no. 3, 1455-1519. MR 2661534

46. Lev Rozansky and Edward Witten, Hyper-Kähler geometry and invariants of three-manifolds, Selecta Math. (N.S.) 3 (1997), no. 3, 401-458. MR 1481135 (98m:57041)

47. Boris Shoikhet, On the Duflo formula for $L_{\infty}$-algebras and Q-manifolds, arXiv:math/9812009 (1998).

48. Jim Stasheff, Constrained Poisson algebras and strong homotopy representations, Bull. Amer. Math. Soc. (N.S.) 19 (1988), no. 1, 287-290. MR 940489 (90a:58057)

49. Kyousuke Uchino, Derived brackets and sh Leibniz algebras, J. Pure Appl. Algebra 215 (2011), no. 5, 1102-1111. MR 2747242 (2011m:17006)

50. Izu Vaisman, Remarks about differential operators on foliate manifolds, An. Şti. Univ. "Al. I. Cuza" Iaşi Sect. I a Mat. (N.S.) 20 (1974), no. 2, 327-350. MR 0458448 (56 \#16651)

51. Luca Vitagliano, On the strong homotopy Lie-Rinehart algebra of a foliation, Commun. Contemp. Math. 16 (2014), no. 6, 1450007, 49. MR 3277952

52. _ On the strong homotopy associative algebra of a foliation, Commun. Contemp. Math. 17 (2015), no. 2, 1450026, 34. MR 3313214

53. _ Representations of homotopy Lie-Rinehart algebras, Math. Proc. Cambridge Philos. Soc. 158 (2015), no. 1, 155-191. MR 3300319

54. Yannick Voglaire and Ping Xu, Rozansky-Witten-type invariants from symplectic Lie pairs, Comm. Math. Phys. 336 (2015), no. 1, 217-241. MR 3322372

55. Ping Xu, Quantum groupoids, Comm. Math. Phys. 216 (2001), no. 3, 539-581. MR 1815717 (2002f:17033)

56. Shilin $\mathrm{Yu}$, Dolbeault dga of formal neighborhoods and $L_{\infty}$ algebroids, $\mathrm{PhD}$ thesis, Penn State University, State College, PA, 2013. 
Department of Mathematics, Tsinghua University

E-mail address: zchen@math.tsinghua.edu.cn

Department of Mathematics, Penn State University

E-mail address: stienon@math.psu.edu

Department of Mathematics, Penn State University

E-mail address: ping@math.psu.edu 